\title{
Perturbative Quantum Field Theory via Vertex Algebras
}

\author{
Stefan Hollands ${ }^{1 *}$, Heiner Olbermann ${ }^{1 \dagger}$, \\ ${ }^{1}$ School of Mathematics, Cardiff University, UK
}

29 June 2009

\begin{abstract}
In this paper, we explain how perturbative quantum field theory can be formulated in terms of (a version of) vertex algebras. Our starting point is the WilsonZimmermann operator product expansion (OPE). Following ideas of a previous paper [arXiv:0802.2198], we consider a consistency (essentially associativity) condition satisfied by the coefficients in this expansion. We observe that the information in the OPE coefficients can be repackaged straightforwardly into "vertex operators" and that the consistency condition then has essentially the same form as the key condition in the theory of vertex algebras. We develop a general theory of perturbations of the algebras that we encounter, similar in nature to the Hochschild cohomology describing the deformation theory of ordinary algebras. The main part of the paper is devoted to the question how one can calculate the perturbations corresponding to a given interaction Lagrangian (such as $\lambda \varphi^{4}$ ) in practice, using the consistency condition and the corresponding non-linear field equation. We derive graphical rules, which display the vertex operators (i.e., OPE coefficients) in terms of certain multiple series of hypergeometric type.
\end{abstract}

\section{Introduction}

It is becoming increasingly clear that Quantum Field Theory (QFT) is not only a very successful theoretical formalism to describe a variety of physical situations such as elementary particle collisions or critical phenomena, but also a highly interesting and complex mathematical structure. This manifests itself not least in the range of different mathematical

\footnotetext{
*HollandsS@Cardiff.ac.uk
}

†OlbermannH@Cardiff.ac.uk 
disciplines that come to play an important role in this theory, such as functional analysis, combinatorics, complex analysis, algebra, geometry, measure theory etc.

Not surprisingly, there exist correspondingly many approaches to QFT, such as the pathintegral approach, the approach via diagrammatic/perturbative expansions, algebraic approaches, stochastic quantization, axiomatic approaches, and more. These approaches are, at some level, known to be equivalent, but each one of them seems to have different weaknesses and strengths. In a recent paper [14] (see also [16] for a related proposal), one of us proposed a new approach of an algebraic nature that is based on elevating the KadanoffWilson-Zimmermann operator product expansion (OPE) $[29,30]$ to the status of a fundamental structure. The operator product expansion is traditionally viewed as a property of the Schwinger functions of the composite fields of the theory. It can be stated as saying that

$$
\left\langle\mathcal{O}_{a}(x) \mathcal{O}_{b}(0) \prod_{i} \mathcal{O}_{d_{i}}\left(y_{i}\right)\right\rangle \sim \sum_{c} C_{a b}^{c}(x)\left\langle\mathcal{O}_{c}(0) \prod_{i} \mathcal{O}_{d_{i}}\left(y_{i}\right)\right\rangle
$$

Here, $x \in \mathbb{R}^{D}$ is a point in Euclidean space not equal to 0 , the $y_{i}$ are distinct points with $\left|y_{i}\right|>|x|$, and $a, b, c$ etc. are indices labeling the composite fields of the theory, which in turn are monomials in the basic field and its derivatives. The OPE-coefficients $C_{a b}^{c}$ are distributions that are independent of the "spectator fields" $\mathcal{O}_{d_{i}}\left(y_{i}\right)$, whereas the brackets indicate the Schwinger functions of the (Euclidean) QFT under consideration. They may be constructed e.g. by giving precise mathematical sense to a functional integral of the type

$$
\left\langle\prod_{i} \mathcal{O}_{a_{i}}\left(x_{i}\right)\right\rangle=\lim _{\Lambda \rightarrow \infty} \int \prod_{i} \mathcal{O}_{a_{i}}\left(x_{i}\right) \exp \left[-S_{\Lambda}(\varphi)\right] \mathrm{d} \mu_{\Lambda}(\varphi) .
$$

either in perturbation theory $[21,22]$, or non-perturbatively, see e.g. [5, 18, 25, 23]. In the above formula, $\mathrm{d} \mu_{\Lambda}(\varphi)$ is a Gaussian measure associated with the free part of a classical action, and $S_{\Lambda}$ is the full action including all counterterms, depending on the cutoff scale $\Lambda$. The OPE coefficients characterize the short-distance properties $(|x| \rightarrow 0)$ of the Schwinger functions. The formula is normally understood as an asymptotic expansion in this limit, with coefficients $C_{a b}^{c}$ becoming more and more smooth in their argument as the dimension of the operator indicated by $\mathcal{O}_{c}$ increases.

The proposal of $[14,16]$ was to view the OPE coefficients not as a secondary structure, but as the defining structure of the theory - some kind of "structure functions" of a suitable algebraic structure governing the field theory. In order to display this algebraic structure, we looked at Schwinger functions with a triple insertion $\mathcal{O}_{a}(x) \mathcal{O}_{b}(y) \mathcal{O}_{c}(0)$, assuming that $0<|x-y|<|y|<|x|$. It is then plausible that one obtains equivalent results when performing successive OPE's in the following two alternative ways indicated by the different positioning of the parenthesis in $\left(\mathcal{O}_{a}(x) \mathcal{O}_{b}(y)\right) \mathcal{O}_{c}(0)=\mathcal{O}_{a}(x)\left(\mathcal{O}_{b}(y) \mathcal{O}_{c}(0)\right)$. For example, the left side means that we perform an OPE of the fields $\mathcal{O}_{b}(y)$ with the field $\mathcal{O}_{c}(0)$, and a subsequent $\mathrm{OPE}$ of the result with $\mathcal{O}_{a}(x)$. The equality sign between both sides means that 
the results should be the same when inserted in a Schwinger function as above. In terms of the OPE-coefficients this means that we expect the relation

$$
\sum_{d} C_{a b}^{d}(x-y) C_{d c}^{e}(y)=\sum_{d} C_{b c}^{d}(y) C_{a d}^{e}(x) .
$$

We called this the "consistency" or "associativity" condition in [14]. We observe that this relation becomes much more transparent if we introduce the following notation: first, we introduce an abstract infinite dimensional complex vector space, $V$, whose basis elements $a \in V$ are in one-to-one correspondence with the composite fields $\mathcal{O}_{a}$ of the theory. Thus, by a slight abuse of notation, we use the labels $a, b, c$ both as labels of the fields, and simultaneously for the basis elements of $V$. We also define the corresponding (algebraic) dual vector space $V^{*}$. We then define a "vertex operator" $Y(a, x): V \rightarrow V$ as the endomorphism of $V$ whose matrix elements are given by

$$
\langle c|Y(a, x)| b\rangle:=C_{a b}^{c}(x)
$$

where we use the usual physicist's notation for basis vectors of $V$ as "kets" $|a\rangle$ and the corresponding dual basis vectors of $V^{*}$ as "bras" $\langle b|$. With this notation, the consistency condition reads simply

$$
Y(a, x) Y(b, y)=Y(Y(a, x-y) b, y),
$$

which holds again when $|x|>|y|>|x-y|>0$. In this form, our condition is almost identical in appearance to (one form of) the key relation in the theory of vertex operator algebras $[19,3,9,11]$, hence the name "vertex operator" for $Y$. We explain the relationship of our approach to vertex algebras in somewhat more detail in sec. 6 of this paper, but we emphasize from the outset that our setup is intended to be much more general than that usually encountered in this theory; for example, we do not assume that the theory is conformally invariant, and we admit general dimensions $D$, even though we expect nontrivial examples only in $D \leq 4$. As a consequence, the contents of our consistency condition and that in vertex algebras are actually rather different despite the similar appearance. An alternative treatment of the free field vertex algebra in $D$ dimensions can already be found in [24] and [4].

The main purpose of the paper is to outline how the standard constructions in QFT, such as perturbative expansion, BRST cohomology etc. can be formulated in terms of the vertex operators $Y$, and what new viewpoint one thereby gets for these constructions. As we will see, if we combine the consistency condition with a perturbative expansion, and with the non-linear field equations corresponding to the classical action $S_{\Lambda}$ in the path integral above, then we obtain in a new scheme for doing perturbative calculations. In more detail, the contents of the paper are as follows:

- In sec. 2 we begin by explaining the general setup. In particular, we explain in abstract terms how ordinary perturbation theory fits into the framework of vertex algebras. 
As we show translating constructions of [14] into the framework of vertex algebras, one can define a cohomology of "Hochschild-type" associated with a $D$-dimensional vertex algebra (in our sense), and perturbations may be characterized in terms of this cohomology. We also explain how the framework may be generalized to also include the BRST-construction for gauge theories.

- In sec. 3, we explain how the vertex algebra of the $D$-dimensional free massless field can be obtained from the Schwinger functions, and we thereby obtain a concrete (albeit somewhat trivial) example for our setting. This section also serves as the starting point to the later sections.

- In sec. 4, we then construct the deformations ("perturbations") of the free field vertex algebra corresponding to an interaction term such as $\lambda \varphi^{4}$ in the classical Lagrangian. We show how one can obtain a closed form expression for the $i$-th order deformation of the vertex operators based on the repeated use of the consistency condition and the (non-linear) field equation [see eqs. (5.114) and (5.111) for the final form of these expressions]. The case $D=2$ is in many ways simpler than the case $D>2$, so we discuss it separately. However, in both cases, the final result is expressed in terms of sums that are associated with certain tree graphs and associated loop graphs. The resulting sums and integrals involve Gegenbauer/Legendre functions and are reminiscent of the expressions one obtains using the "Gegenbauer polynomial $x$-space technique" of [6] for multi-loop Feynman diagrams.

- In sec. 5, we discuss an alternative representation of the deformed vertex operators in terms of certain multiple infinite sums. These sums have a similarity with the hypergeometric series, and we believe that this opens up an interesting connection between our vertex algebras and a class of special functions.

- In sec. 6 we discuss in some detail the similarities and differences between our notion of vertex algebra and notions that were previously given in the context of 2-dimensional CFT's.

- Various results and definitions related to Legendre functions in $D$-dimensions needed in the main text are given in app. A and B.

This paper is about the mathematical structure of QFT, but it is by itself not completely mathematically rigorous. We analyze the structure and exploit the consequences of the consistency condition in a mathematically rigorous way, but we do not justify the consistency condition itself (apart from free field theory) and rather treat it as a hypothesis. In this sense, our approach can be thought of as some sort of "bootstrap". In order to justify the consistency condition, one can e.g. start from the OPE of the Schwinger functions, and show that performing the expansion of a triple product of fields 
lead to the same result, as we described. We will come back to this issue in another paper [15].

Notations and conventions: $x, y$ etc. denote points in $\mathbb{R}^{D}$, with scalar product $x \cdot y=$ $\sum_{\mu} x_{\mu} y_{\mu}$, and norm $r^{2}=|x|^{2}=x \cdot x$. We also use the notation $\hat{x}:=x / r \in S^{D-1}$ for the angular part if $x \neq 0 . \mathrm{P}(z, \nu, D)$ denote the Legendre functions, see appendix $\mathrm{A}$, and $\psi=$ $\Gamma^{\prime} / \Gamma$ is the Psi-function. The surface area of the $(D-1)$-dimensional sphere is abbreviated $\sigma_{D}=\frac{2 \pi^{D-2}}{\Gamma(D / 2)}$ and $K_{D}=\sqrt{D-2}$. The natural numbers $\mathbb{N}$ include $0, \mathbb{N}=\{0,1,2, \ldots\}$.

\section{General setup}

In the previous section, we explained the basic idea that connects QFT as formulated in terms of Schwinger functions with vertex operators $Y(a, x)$ satisfying a consistency condition. Let us isolate the properties that we expect these vertex operators to have, reflecting the general known properties of the Schwinger functions:

1. First, we state formally that there is an identity operator in field theory. This is a distinguished element $\mathbf{1} \in V$. The OPE of $\mathbf{1}$ with any other field is trivial and the vertex operator associated with $\mathbf{1}$ is hence given by $Y(\mathbf{1}, x)=i d$, where $i d$ is the identity on $V$.

2. We have already said that the OPE coefficients are in general expected to be distributions on $\mathbb{R}^{D}$. In Euclidean space, the situation is actually better and the coefficients are analytic except for the origin. Thus, if we denote by $\mathcal{A}\left(\mathbb{R}^{D} \backslash\{0\}\right)$ the set of such analytic functions then we expect that $Y(a,.) \in \mathcal{A}\left(\mathbb{R}^{D} \backslash\{0\}\right) \otimes \operatorname{End}(V)$.

3. The Schwinger functions have an obvious invariance property under the Euclidean group $S O(D)$ (or its covering group, if there are spinor fields in the theory). These invariance properties are inherited by the OPE coefficients, and we hence have corresponding invariance properties of the vertex operators: $Y(a, g \cdot x)=$ $R(g) Y\left(R(g)^{-1} a, x\right) R(g)^{-1}, g \in S O(D)$, for some representation $R$ of $S O(D)$ on $V$. Note, however, that there is no similar covariance under translations, as the vertex operator in effect depend on the choice of the origin $0 \in \mathbb{R}^{D}$ as the reference point in the OPE.

4. The key condition is the consistency condition, which we have already motivated and which we repeat:

$$
Y(a, x) Y(b, y)=Y(Y(a, x-y) b, y),
$$

for all $a, b \in V$ and all $x, y \in \mathbb{R}^{D}$ subject to $0<|x-y|<|y|<|x|$. Note that there is an implicit statement about the convergence of an infinite sum made here: If we apply e.g. the right side of the equation to a vector $c \in V$, then we effectively state that $Y(b, y) c$ is in the domain of $Y(a, x)$. When written in a standard basis of composite 
fields, the vector $d=Y(b, y) c$ typically is an infinite linear combination of such fields even if $b, c$ are from a basis of composite fields. Thus, $Y(a, x) d$ will also be an infinite sum when written in the basis of composite fields, and we require, in effect, that this sum converges. As we discuss in more detail in sec. 6, there is no such issue in the usual formulation of vertex algebras, where all sums are either formal, or finite.

The Schwinger functions have other properties that one can readily translate into properties of the vertex operators, such as (anti-) symmetry properties under the exchange of fields, scaling properties, hermiticity properties, etc. To keep the discussion transparent at this point, we will not go into this here, but state some of these conditions as we go along.

In renormalized perturbation theory, one is naturally led to consider field-redefinitions, for example when considering changes in the renormalization scheme. A field redefinition is simply a linear transformation which maps a quantum field to a linear combination of quantum fields, and thereby gives a corresponding transformation of the Schwinger functions. Such field redefinitions change in an evident way also the OPE coefficients of the theory. In terms of the vertex operators, a field redefinition is simply an invertible complex linear map $Z: V \rightarrow V$ which commutes with $R(g)$ for all $g \in S O(D)$, and which satisfies $Z \mathbf{1}=\mathbf{1}$. The OPE coefficients associated with the transformed fields give rise to a correspondingly transformed vertex operator, given by

$$
Y^{\prime}(a, x)=Z Y(x, Z a) Z^{-1}
$$

This vertex operator satisfies the same properties as above, and will be considered "equivalent".

An important construction in QFT is to consider perturbations of a given theory. The idea is usually to start from a free field theory with explicitly known Schwinger functions/OPE and to construct perturbations of these quantities, order by order in a "small" parameter $\lambda$ characterizing the size of the perturbation. In the path integral, one expands $\exp \left(-S_{\Lambda}\right)$ in terms of this parameter, and then performs the Gaussian integral for each term in this expansion. Such a procedure will also give rise to a corresponding expansion of the OPE coefficients, see e.g. [20], and thereby to an expansion of the vertex operators of the form

$$
Y(a, x)=\sum_{i=0}^{\infty} \lambda^{i} Y_{i}(a, x) .
$$

The zeroth order term $Y_{0}(a, x)$ of this - in general only formal-expansion corresponds to the free field theory around which one expands. The higher terms represent the perturbations. Because the full $Y(a, x)$ must satisfy the properties 1) -4) stated above for all $\lambda$ (or at least in the sense of formal perturbation series), the expansion coefficients $Y_{i}(a, x)$ must satisfy analogous properties. The most prominent one is the consistency condition, which at $i$-th order reads

$$
\sum_{j=0}^{i} Y_{j}(a, x) Y_{i-j}(b, y)=\sum_{j=0}^{i} Y_{j}\left(Y_{i-j}(a, x-y) b, y\right) \text {. }
$$


It is satisfied at order $i=0$, because one can prove it for a free field theory. As already pointed out in [14], there is an interesting cohomological interpretation of eq. (2.9), and we now briefly explain how this comes about. Let $n \geq 0$ and define $\Omega^{n+1}(V)$ to be a space of linear maps

$$
f_{n}\left(x_{1}, \ldots, x_{n}\right): V \otimes \cdots \otimes V \rightarrow \operatorname{End}(V)
$$

that are defined for certain configurations $\left(x_{1}, \ldots, x_{n}\right) \in \mathbb{R}^{n D}$ of mutually different points, and where there are $n$ tensor copies of $V$. More precisely, $f_{n}$ 's should be real analytic functions of $x_{1}, \ldots, x_{n} \in \mathbb{R}^{n D}$ in the open domain

$$
D_{n}=\left\{\left(x_{1}, \ldots, x_{n}\right) \in \mathbb{R}^{n D}: r_{1, i-1}<r_{i-1, i}<r_{i-2, i}<\ldots<r_{1, i}\right\},
$$

where $r_{i, j}=\left|x_{i}-x_{j}\right|$, taking values in $\operatorname{End}\left(V^{\otimes n}, \operatorname{End}(V)\right)$. Next, we define from $Y_{0}$ a linear operator $b: \Omega^{n}(V) \rightarrow \Omega^{n+1}(V)$ by the formula

$$
\begin{aligned}
& \left(b f_{n}\right)\left(x_{1}, \ldots, x_{n+1} ; a_{1}, \ldots, a_{n+1}\right):=Y_{0}\left(a_{1}, x_{1}\right) f_{n}\left(x_{2}, \ldots, x_{n+1} ; a_{2}, \ldots, a_{n+1}\right) \\
+ & \sum_{i=1}^{n}(-1)^{i} f_{n}\left(x_{1}, \ldots, \widehat{x}_{i}, \ldots, x_{n+1} ; a_{1}, \ldots, Y_{0}\left(a_{i}, x_{i}-x_{i+1}\right) a_{i+1}, \ldots a_{n+1}\right) \\
+ & (-1)^{n+1} f_{n}\left(x_{1}, \ldots, x_{n} ; a_{1}, \ldots, a_{n}\right) Y_{0}\left(a_{n+1}, x_{n+1}\right) .
\end{aligned}
$$

This linear operator is presumably not defined on all such $f_{n}$, but only on a certain domain of definition, but we ignore this issue here for simplicity. We formally calculate [14] using the consistency condition for $Y_{0}$ that

$$
b\left(b f_{n}\right)=0
$$

i.e., $b$ is a coboundary operator. The domains $D_{n}$ are needed in order to be able to apply the consistency condition. We denote by

$$
H^{n}\left(V, Y_{0}\right):=\left\{\operatorname{ker} b: \Omega^{n} \rightarrow \Omega^{n+1}\right\} /\left\{\operatorname{ran} b: \Omega^{n-1} \rightarrow \Omega^{n}\right\}
$$

the $n$-th cohomology ring of the chain complex $\left(\oplus_{n} \Omega^{n}(V), b\right)$.

The connection to the consistency condition eq. (2.9) at $i$-th order in perturbation theory now arises as follows: At $i=1$, the equation can be restated as simply saying that

$$
b Y_{1}=0 .
$$

If $Y_{1}$ arises from $Y_{0}$ by merely a field redefinition (2.7) with $Z=\sum_{i=1}^{n} z_{i} \lambda^{i}$, then this means

$$
Y_{1}=b z_{1} .
$$

Consequently, the non-trivial first order perturbations may be viewed as elements in $H^{2}\left(V, Y_{0}\right)$, and hence such perturbations only exist if this space is non-trivial. Continuing in this way, the condition (2.9) at $i$-th order can be stated as saying that

$$
b Y_{i}=w_{i}
$$


where $w_{i} \in \Omega^{3}(V)$ is defined by the terms in eq. (1.3) with $j \neq 0, j \neq i$. It can be checked inductively [14] that $b w_{i}=0$, so $\left[w_{i}\right]$ defines a class in $H^{3}\left(V, Y_{0}\right)$. Evidently, the $i$-th order perturbation $Y_{i}$ exists, if, in fact, $w_{i} \in \operatorname{ran} b$, i.e. if it defines the zero class in $H^{3}\left(V, Y_{0}\right)$. The freedom of choosing different solutions to eq. (2.17) corresponds to elements in $H^{2}\left(V, Y_{0}\right)$. In this way, we get an interpretation of perturbation theory in terms of the cohomology rings $H^{n}\left(V, Y_{0}\right)$.

There is no difficulty to extend this framework to more complicated situations where one has additional symmetries that one would like to preserve in the deformation process. Suppose e.g. that the free theory is a free $U(1)^{N}$ gauge theory with associated ghosts. Then in the free field theory we have an associated free BRST-operator $s_{0}$ with the property $s_{0}^{2}=0$ and $s_{0} \circ g h=(g h-i d) \circ s_{0}$, where $g h$ is the "ghost number", i.e. a linear operator on $V$ with integer spectrum that provides a corresponding grading. This acts on the composite fields of the theory, and hence, as a linear transformation $s_{0}: V \rightarrow V$ satisfying $\gamma s_{0}+s_{0} \gamma=0$, where $\gamma: V \rightarrow V$ is a $\mathbb{Z}_{2}$-grading of $V$ that corresponds to the Bose/Fermi character of the fields. The concrete form of $s_{0}$ in terms of the gauge field and ghost/auxiliary fields is given in many textbooks, see e.g. [31]. To explain our general scheme it is only important to know that the BRST-invariance of the Schwinger functions of the free gauge theory gives rise to a corresponding invariance of the OPE, namely, the OPE of BRST-invariant fields again contains only such fields. In terms of the vertex operators, this is expressed by the relation

$$
s_{0} Y_{0}(a, x)+Y_{0}(\gamma a, x) s_{0}+Y_{0}\left(s_{0} a, x\right)=0 .
$$

We would now like to ask whether it is possible to find a deformation of $Y_{0}$ as above to a $Y$, and a corresponding deformation of $s_{0}$ to an $s$

$$
s=\sum_{i=0}^{\infty} s_{i} \lambda^{i}
$$

such that $s^{2}=0$, i.e.

$$
0=\sum_{j=0}^{i} s_{j} s_{i-j}
$$

and such that eq. (2.18) continues to hold for $Y, s$, that is, at $i$-th order,

$$
\sum_{j=0}^{i} s_{j} Y_{i-j}(a, x)+Y_{j}(\gamma a, x) s_{i-j}+Y_{j}\left(s_{i-j} a, x\right)=0 .
$$

The problem of finding simultaneously the $s_{i}$ and $Y_{i}$ subject to eqs. (2.21), (2.20) and (2.9) is again of a cohomological nature. To set up the cohomology ring in question, we extend the action of $s_{0}$ from $V$ to $\Omega^{n}(V)$ by setting

$$
\begin{aligned}
\left(B f_{n}\right)\left(x_{1}, \ldots, x_{n} ; a_{1}, \ldots, a_{n}\right) & :=s_{0} f_{n}\left(x_{1}, \ldots, x_{n} ; a_{1}, \ldots, a_{n}\right) \\
& +\sum_{j=1}^{n} \gamma f_{n}\left(x_{1}, \ldots, x_{n} ; a_{1}, \ldots, a_{j-1}, \gamma s_{0} a_{j}, \gamma a_{j+1}, \ldots, \gamma a_{n}\right)
\end{aligned}
$$


Then one verifies [14] that $B^{2}=0$ using $s_{0}^{2}=0$ and that $b B+B b=0$ using eq. (2.18), i.e. $B$ is another coboundary operator which is compatible with $b$. In the language of differential complexes, $(b, B)$ gives rise to a double complex $\left(\oplus_{n, g} \Omega^{n, g}(V), b, B\right)$, whose cohomology rings are denoted $H^{n, g}\left(V, Y_{0}, s_{0}\right)$. Here $n$ is as above, and $g$ corresponds to the grading of $V$ (and correspondingly the spaces $\left.\Omega^{n}(V)=\oplus_{g} \Omega^{n, g}(V)\right)$ by the ghost number $g h$. We have the following commutative diagram:

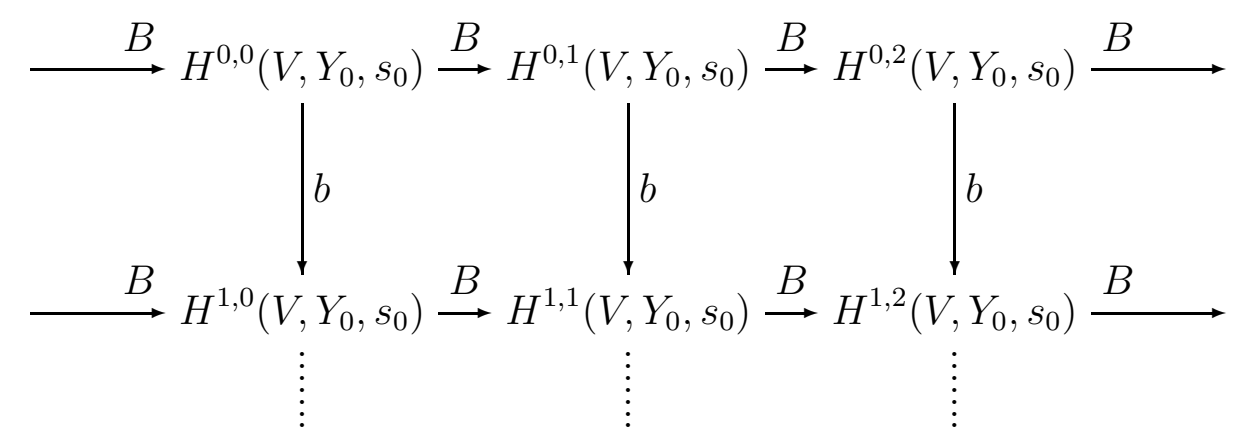

As is standard in this situation, we may form the total complex whose cohomology rings are given by

$$
H^{m}\left(V, Y_{0} \mid s_{0}\right)=\bigoplus_{n+g=m} H^{n, g}\left(V, Y_{0}, s_{0}\right),
$$

and whose total coboundary operator is $B+b$, i.e. $(B+b)^{2}=0$. The direct sum in eq. (2.23) consists of the cohomology groups lined up on the diagonal running from $H^{m, 0}\left(V, Y_{0}, s_{0}\right)$ to $H^{0, m}\left(V, Y_{0}, s_{0}\right)$ in the above diagram.

If we form $\alpha_{i}=\left(s_{i}, Y_{i}, 0,0, \ldots\right)$, then the combined condition for the first order perturbation $\alpha_{1}$ arising from the first order consistency condition and the first order BRST-invariance can be written as

$$
(b+B) \alpha_{1}=0,
$$

while the condition for the first order perturbations to be due to a first order field redefinition $\zeta_{i}=\left(z_{i}, 0,0, \ldots\right)$ is written as

$$
\alpha_{1}=(B+b) \zeta_{1} .
$$

Thus, first order perturbations of the BRST operator and vertex operators are given by a class $\left[\alpha_{1}\right] \in H^{2}\left(V, Y_{0} \mid s_{0}\right)$. Likewise, the conditions for the $i$-th order perturbations $\alpha_{i}$ can be written as

$$
(B+b) \alpha_{i}=\beta_{i},
$$

where $\beta_{i}$ is calculable from the $\alpha_{j}, j \leq i-1$, and where one can compute $(B+b) \beta_{i}=0$. Thus, the potential $i$-th order obstruction is the class $\left[\beta_{i}\right] \in H^{3}\left(V, s_{0} \mid Y_{0}\right)$. The details of this analysis are completely analogous to [14]. 


\section{The free field OPE vertex algebra}

In this section, we illustrate our abstract framework for the OPE in a simple example. Our example is the free quantum field theory obeying the linear field equation

$$
\Delta \varphi=0 .
$$

The space $V$ of fields in this theory may be taken to be the unital, free, commutative ring generated by the identity $\varphi$ and its derivatives. In other words, the elements of $V$ are in one-to-one correspondence with monomials in $\partial_{\mu_{1}} \ldots \partial_{\mu_{k}} \varphi$, and $\partial_{\mu}, \mu=1, \ldots, D$ are the derivations that act as if they were ordinary partial derivatives. To implement the field equation, we simply set to zero any expressions containing a factor the form $\delta^{\mu_{i} \mu_{j}} \partial_{\mu_{1}} \ldots \partial_{\mu_{k}} \varphi$, i.e., monomials that would vanish if $\varphi$ was an actual field satisfying the field equation. Because monomials containing a trace of $\partial_{\mu_{1}} \ldots \partial_{\mu_{k}} \varphi$ are set to zero, $V$ is spanned by all trace-free monomials. Thus, if we denote by curly brackets $t_{\left\{\mu_{1} \ldots \mu_{k}\right\}}$ the trace-free part of a symmetric tensor, then a basis of $V$ is given by $\mathbf{1}$, together with the set of monomials of the form $\prod \partial_{\left\{\mu_{1}\right.} \cdots \partial_{\left.\mu_{k}\right\}} \varphi$.

It is convenient for latter purposes to choose a particular basis. For this, we consider the space of harmonic polynomials in $D$ real variables homogeneous of degree $l$, i.e. the set of all polynomials $h(x)$ in $D$ variables $\left(x \in \mathbb{R}^{D}\right)$ with complex coefficients satisfying $h(t x)=t^{l} h(x)$, and $\Delta h(x)=0$. Some relevant facts about such polynomials are collected in appendix A. We denote by $h_{l, m}, m=1, \ldots, N(D, l)$ a basis of degree $l$ harmonic polynomials. (The number of linearly independent polynomials of this kind, $N(D, l)$, can be found in appendix A.) We normalize this basis so that ${ }^{1}$

$$
\int_{S^{D-1}} \bar{h}_{l, m}(\hat{x}) h_{l^{\prime}, m^{\prime}}(\hat{x}) \mathrm{d} \Omega(\hat{x})=\delta_{l, l^{\prime}} \delta_{m, m^{\prime}}
$$

where $\mathrm{d} \Omega$ is the standard integration element on the sphere, and $\hat{x}=x /|x|$. A basis of $V$ is then given by $\mathbf{1}$, together with the elements

$$
a=\prod_{l, m} \frac{1}{\sqrt{a_{l, m} !}}\left(c_{l}^{-1} \bar{h}_{l, m}(\partial) \varphi\right)^{a_{l, m}},
$$

where $a=\left\{a_{l, m} \in \mathbb{N} \mid l \geq 0, m=1, \ldots, N(l, D)\right\}$ is identified at the same time with a vector in $V$ and a multi-index of non-negative integers, only finitely many of which are non-zero, and $c_{l}$ is given in appendix C. The Schwinger functions of the model are well-known. For $2 s$ factors of the basic field they are given by $(D>2)$

$$
\left\langle\prod_{i=1}^{2 s} \varphi\left(x_{i}\right)\right\rangle=\sum_{\sigma \in \operatorname{Sym}(2 s)} \prod_{j=1}^{s}\left|x_{\sigma(2 j-1)}-x_{\sigma(2 j)}\right|^{2-D}
$$

\footnotetext{
${ }^{1}$ With this normalization, the harmonic polynomials restricted to $S^{D-1}$ are the $D$-1-dimensional spherical harmonics.
} 
for non-coincident $x_{i} \in \mathbb{R}^{D}$. For $2 s+1$ factors of the basic field the Schwinger function is zero. Partial derivatives can be taken on both sides of the equation to get the Schwinger functions of all $\varphi$-linear fields. The Schwinger functions of composite operators can be obtained in the same manner, writing a composite field as a product of $\varphi$-linear fields with the same argument $x \in \mathbb{R}^{D}$ and omitting permutations $\sigma$ with $x_{\sigma(2 j-1)}=x_{\sigma(2 j)}$ for some $j \in\{1, \ldots, s\}$ in eq. (3.30). For example, if none of the composite field has derivatives, we have

$$
\left\langle\prod_{v=1}^{n} \varphi^{p_{v}}\left(x_{v}\right)\right\rangle=\sum_{\text {graphs } G} \prod_{e=(v w) \in G}\left|x_{v}-x_{w}\right|^{2-D} .
$$

Here, the sum is over all graphs with coordination numbers $\left\{p_{v}\right\}$, and the product is over all edges $e=(v w)$ of the graph. For composite fields with derivatives, one has a similar formula. From the Schwinger functions, one gets the OPE coefficients according to eq. (1.1), and from the OPE coefficients, one gets the $Y_{0}(a, x)$ according to eq. (1.4). In $D=2$ dimensions, the propagators are replaced by $\ln \left|x_{v}-x_{w}\right|$. We now present the results of these calculations, leaving the details to appendix C. The subscript " 0 " reminds us that we are dealing with a free field in this section.

In order to present explicitly the $Y_{0}$ 's, it is convenient to view $V$ as a "Fock-space," with $a_{l, m}$ (see eq. (3.29)) interpreted as the "occupation number" of the "mode" labeled by $l, m$, and with 1 playing the role of "Fock-vacuum" denoted $|0\rangle$ (vanishing occupation number). On this Fock-space, one can then define creation and annihilation operators $\mathbf{b}_{l, m}, \mathbf{b}_{l, m}^{+}: V \rightarrow$ $V$, see appendix C. They satisfy the standard commutation relations

$$
\left[\mathbf{b}_{l, m}, \mathbf{b}_{l^{\prime}, m^{\prime}}^{+}\right]=\delta_{l, l^{\prime}} \delta_{m, m^{\prime}} i d, \quad\left[\mathbf{b}_{l, m}^{+}, \mathbf{b}_{l^{\prime}, m^{\prime}}^{+}\right]=\left[\mathbf{b}_{l, m}, \mathbf{b}_{l^{\prime}, m^{\prime}}\right]=0
$$

where $i d$ is the identity operator on $V$. In this language, the basis elements of $V$ are written as

$$
a=\prod_{l, m} \frac{\left(\mathbf{b}_{l, m}^{+}\right)^{a_{l, m}}}{\sqrt{a_{l, m} !}}|0\rangle .
$$

We now give the formula for $Y_{0}(\varphi, x)$ corresponding to the basic field. For $D>2$, this is given by

$$
\begin{aligned}
& Y_{0}(\varphi, x)=K_{D} r^{-(D-2) / 2} \sum_{l=0}^{\infty} \sum_{m=1}^{N(l, D)} \frac{1}{\sqrt{\omega(D, l)}} \times \\
& {\left[r^{l+(D-2) / 2} h_{l, m}(\hat{x}) \mathbf{b}_{l, m}^{+}+r^{-l-(D-2) / 2} \overline{h_{l, m}(\hat{x})} \mathbf{b}_{l, m}\right], }
\end{aligned}
$$

where $K_{D}=\sqrt{D-2}$, and the "frequency" $\omega(l, D)$ is given by $2 l+D-2$, see appendix C. For a general $a \in V$ of the form eq. (3.29), the vertex operators $Y_{0}(a, x): V \rightarrow V$ are

$$
Y_{0}(a, x)=: \prod_{l, m} \frac{1}{\left(a_{l, m} !\right)^{1 / 2}}\left\{c_{l}^{-1} h_{l, m}(\partial) Y_{0}(\varphi, x)\right\}^{a_{l, m}}: \text {. }
$$


Here, double dots : $\cdots$ : mean "normal ordering", i.e., all creation operators are to the right of all annihilation operators.

It can be checked explicitly that the $Y_{0}$ satisfy the properties 1$)$-4). The representation $R: S O(D) \rightarrow \operatorname{End}(V)$ in 3$)$ is characterized by

$$
R(g) \mathbf{b}_{l, m}^{+} R(g)^{-1}=\sum_{m^{\prime}=1}^{N(D, l)} \mathcal{D}_{m, m^{\prime}}^{l}(g) \mathbf{b}_{l, m^{\prime}}^{+},
$$

where $\mathcal{D}^{l}$ in turn is the representation ${ }^{2}$ of $S O(D)$ on the space of degree $l$ harmonic polynomials, i.e. $\sum_{m^{\prime}} \mathcal{D}_{m, m^{\prime}}^{l}(g) h_{l, m^{\prime}}(x)=h_{l, m}(g \cdot x)$. In order to demonstrate the consistency condition 4), one must make use of the identities related to the harmonic polynomials given in appendix A. We are not going to go through this lengthy calculation here.

\section{Perturbative OPE vertex algebras}

In the previous section we have described the (well-known) OPE of the free, massless bosonic field in $D$ dimensions. The new viewpoint was to understand this as a vertex algebra. Of course, free field theories are only of limited interest, and we therefore now look at perturbations of the free theory. Normally, perturbations are characterized by an interaction Lagrangian, which, together with appropriate counterterms, is inserted into the path integral (1.2) in order to obtain the perturbation series of the Schwinger functions. To make this work, one first considers a regulated path integral, with a regulated interaction, $S_{\Lambda}$, and one then removes the regulator $\Lambda$. This procedure is explained in many textbooks, see e.g. [31], and it leads also to the definition of the OPE coefficients, $C_{a b}^{c}$, see e.g. [20] for a derivation using the Wilson-Wegner-Polchinski RG-flow equations.

As we have explained, in this paper, we want to pursue an approach wherein the OPE is elevated to the status of a fundamental relation, and we should therefore also have a method to calculate the perturbations of the OPE coefficients - or equivalently the $Y$ 's directly, without recourse to the Schwinger functions. In principle, we have outlined how this works in sec. 2. But for this we would need to understand more explicitly the -rather abstractly defined - cohomology rings $H^{2}\left(V, Y_{0}\right)$ and $H^{3}\left(V, Y_{0}\right)$. We have so far been unable to do so. However, one would think that perturbations should also be describable in terms of an interaction Lagrangian, in the same way as in the usual approach, because one is talking about the same theory after all.

We now explain one way how to proceed. Let us assume that the "bare interaction" (in the usual QFT parlance) is given by a polynomial $\lambda P(\varphi)=\lambda \sum \frac{c_{p}}{p !} \varphi^{p}$. In order to get a well-defined perturbative definition of the Schwinger functions, we also assume that the

\footnotetext{
${ }^{2}$ In $D=3, l$ corresponds to the angular momentum quantum number and $\mathcal{D}^{l}$ is the corresponding irreducible representation. For $D>3$, the representation $\mathcal{D}^{l}$ is reducible.
} 
interaction is renormalizable, i.e. $\operatorname{deg}(P) \frac{D-2}{2} \leq D$. It is a well-known fact in standard perturbation theory that the Schwinger functions of the theory may then be defined so that

$$
\left\langle\left[\Delta \varphi(x)-\lambda P^{\prime}(\varphi(x))\right] \mathcal{O}_{a}(0) \prod_{i} \mathcal{O}_{d_{i}}\left(y_{i}\right)\right\rangle=0
$$

Here, $\mathcal{O}_{a}, \mathcal{O}_{d_{i}}$ are arbitrary composite fields, and the arguments satisfy $\left|y_{i}\right|>|x|>0 .{ }^{3}$ The expression in brackets [...] is of course the non-linear field equation. Let us now apply the OPE to the expression $\left[\Delta \varphi(x)-\lambda P^{\prime}(\varphi(x))\right] \mathcal{O}_{a}(0)$ in the above Schwinger function. Then we get a relation between the OPE coefficients involving $\Delta \varphi$ and those involving $P^{\prime}(\varphi)$. In terms of the vertex operators this relation is

$$
\Delta Y(\varphi, x)=\lambda Y\left(P^{\prime}(\varphi), x\right)
$$

where we are now viewing $P^{\prime}(\varphi)$ as an element in $V$, the abstract vector space whose elements are in one-to-one correspondence with the composite fields.

Next, we expand the vertex operators in a (formal) perturbation series in $\lambda$ as in eq. (2.8), and this immediately leads to the relation

$$
\Delta Y_{i}(\varphi, x)=Y_{i-1}\left(P^{\prime}(\varphi), x\right)
$$

The evident strategy is now to try to design an iterative scheme from this equation, by calculating the order $i$ vertex operator on the left in terms of the lower order $i-1$ vertex operator on the right, starting inductively with $i=1$, as all vertex operators of order $i-1=0$ have been given in the previous section. However, such a procedure runs into the immediate difficulty that the right side involves the vertex operator associated with the composite field $P^{\prime}(\varphi)$, whereas the left side only gives the vertex operator of the basic field $\varphi$. We must therefore introduce a second induction loop which constructs, iteratively, the vertex operators of an arbitrary $a \in V$ from those of $\varphi$. It is the essential strength of our approach that this is possible, using the consistency condition in perturbative form, see eq. (2.9).

Thus, suppose that we are given $Y_{i}(\varphi, x)$, and all other vertex operators up to order $1, \ldots, i-1$. How to find $Y_{i}\left(\varphi^{2}, x\right), Y_{i}\left(\varphi^{3}, x\right)$, etc.? Consider points such that $0<|x-y|<$ $|y|<|x|$ and the following special case of the $i$-th order consistency condition:

$$
\sum_{j=0}^{i} Y_{j}(\varphi, x) Y_{i-j}(\varphi, y)=\sum_{j=0}^{i} Y_{i-j}\left(Y_{j}(\varphi, x-y) \varphi, y\right)
$$

Under the hypothesis that this condition indeed holds for the - yet to be constructed - terms in this equation that are not known at this point, we can draw the following conclusion. Let us look at the term with $j=0$ on the right side of the equation. Using the known form of the $Y_{0}$ 's (from the free theory), we have $Y_{0}(\varphi, x-y) \varphi=|x-y|^{-(D-2)} \mathbf{1}+\varphi^{2}$, plus terms that

\footnotetext{
${ }^{3}$ Otherwise, there would also be "contact terms" in the above equation.
} 
are smooth in $x-y$ and vanish for $x=y$. Using $Y_{i}(\mathbf{1}, y)=0$ for $i>0$, we hence arrive at the relation

$$
Y_{i}\left(\varphi^{2}, y\right)=\sum_{j=0}^{i} Y_{j}(\varphi, x) Y_{i-j}(\varphi, y)-\sum_{j=1}^{i} Y_{i-j}\left(Y_{j}(\varphi, x-y) \varphi, y\right)+\ldots
$$

The dots represent terms that vanish in the limit as $|x-y| \rightarrow 0$. The singular terms with the --signs may be thought of as some sort of "counterterms", which cancel off the divergence of the first term on the right side in the limit. The key thing to note is now that all terms on the right side are known, by induction. Thus, taking the limit, we obtain the desired vertex operator $Y_{i}\left(\varphi^{2}, x\right)$, and, by iterating this procedure in an obvious way, all other $i$-th order vertex operators $Y_{i}\left(\varphi^{3}, x\right), Y_{i}\left(\varphi^{4}, x\right), \ldots$

In summary, our iterative scheme is set up in such a way that, at order $i$, we have to perform one inversion of the Laplace operator in eq. (4.39) to get $Y_{i}(\varphi, x)$, and then we subsequently have to construct all other vertex operators at order $i$ via the consistency condition. Unfortunately, it is not evident from what we have said that the vertex operators constructed in this way will satisfy the consistency condition to all orders. Furthermore, there is certainly the freedom to add to the $i$-th order solution to the inhomogeneous Laplace equation $Y_{i}(\varphi, x)$ a solution to the homogeneous equation. It would seem that both issues are connected, and that one must impose the validity of the $i$-th order consistency condition in order to (partly) fix this ambiguity. One would then, furthermore, expect that the ambiguity is equivalent to the usual sort of renormalization ambiguity, which in our framework is given by eq. (2.7) (with $Z=\sum \lambda^{i} z_{i}$ ) and a change in the coupling constant $\lambda$ by a (formal) diffeomorphism.

We will not address these issues in the present paper but rather focus on the kind of mathematical expressions that one obtains following the above iterative scheme. We divide our discussion into several parts. First, in the next subsection we discuss how to take the inverse of the Laplace operator in a way that is suitable in our setting. Then in subsec. 4.2, we will discuss how to organize the terms that appear in the iteration process by a graphical notation involving trees. In subsec. 4.3, we outline how the "counterterms" may be incorporated into the graphical notation. In sec. 5, we find another representation of the vertex operators in terms of infinite sums of hypergeometric type.

\subsection{Inverting the Laplace operator}

In our inductive scheme, we have to invert the Laplace operator at each step of the induction. According to the general setup, the vertex operators are functions $Y(a,.) \in \mathcal{A}\left(\mathbb{R}^{D} \backslash\{0\}\right) \otimes$ End $(V)$, so we should invert on this function space. However, in perturbation theory, the space of functions is actually much more restricted. At zeroth order, the vertex operators are in fact infinite sums of products of creation/anhihilation operators, harmonic polynomials $h_{l, m}(\hat{x})$ and powers $r^{k}$, where $l \in \mathbb{N}, k \in \mathbb{Z}, 0<m \leq N(D, l)$, see eqs. (3.34) and (3.35). 
Unfortunately, such functions are not stable under the inverse of the Laplace operator - we also get factors of $\ln r$. Inverting again, we get factors of $\ln ^{2} r$, and so on. To incorporate the logarithms, we are thus led to introduce the following spaces of functions for $i \in \mathbb{N}$ :

$$
\mathcal{E}_{i}=\operatorname{span}\left\{r^{k} \ln ^{j} r h_{l, m}(\hat{x}): k \in \mathbb{Z}, j, l \in \mathbb{N}, j \leq i, 1 \leq m \leq N(l, D)\right\} .
$$

Evidently, the union $\cup_{j} \mathcal{E}_{j}$ is a filtered ring $\left(\mathcal{E}_{i} \mathcal{E}_{j} \subset \mathcal{E}_{i+j}\right)$. Any right inverse of the Laplace operator, $G$, takes us between $\mathcal{E}_{i} \rightarrow \mathcal{E}_{i+1}$ for all $i$. Our inductive scheme will hence give us $i$-th order vertex operator in the space

$$
Y_{i}(a, .) \in \mathcal{E}_{i} \otimes \operatorname{End}(V) .
$$

In order to give an explicit formula for $G$ we introduce a residue integral trick for computing right inverses of the Laplacian which we are going to use extensively. We first define

$$
\begin{aligned}
G\left(r^{k} h_{l, m}(\hat{x})\right) & =G\left(\frac{1}{2 \pi \mathrm{i}} \oint_{C} \frac{\mathrm{d} \delta}{\delta} r^{k+\delta} h_{l, m}(\hat{x})\right) \\
& :=\frac{1}{2 \pi \mathrm{i}} \oint_{C} \frac{\mathrm{d} \delta}{\delta} \frac{r^{k+2+\delta}}{(k+2+\delta)(k+D+\delta)-l(l+D-2)} h_{l, m}(\hat{x}) .
\end{aligned}
$$

This defines $G$ as an operator on $\mathcal{E}_{0} \rightarrow \mathcal{E}_{1}$. To extend the trick (4.44) to all of $\mathcal{E}=\cup_{j} \mathcal{E}_{j}$, assume that we are given $f(x) \in \mathcal{E}_{j}$ as a $j$-fold residue integral of the form

$$
f(x)=\prod_{i=1}^{j}\left(\frac{1}{2 \pi \mathrm{i}} \oint_{C_{i}} \frac{r^{\delta_{i}} \mathrm{~d} \delta_{i}}{\delta_{i}}\right) F\left(\delta_{1}, \ldots, \delta_{j}\right) r^{k} h_{l, m}(\hat{x}),
$$

where $F\left(\delta_{1}, \ldots, \delta_{j}\right)$ does not depend on $x$. It is always possible to represent $f \in \mathcal{E}_{j}$ as a linear combination of expressions of the form (4.45), and in fact, this is the form we will encounter. We define the Laplace inverse of $f(x)$ by

$$
\begin{aligned}
G f(x):= & \prod_{i=1}^{j+1}\left(\frac{1}{2 \pi \mathrm{i}} \oint_{C_{i}} \frac{r^{\delta_{i}} \mathrm{~d} \delta_{i}}{\delta_{i}}\right) F\left(\delta_{1}, \ldots, \delta_{j}\right) \\
& \times\left[\left(k+2+\sum_{i=1}^{j+1} \delta_{i}\right)\left(k+D+\sum_{i=1}^{j+1} \delta_{i}\right)-l(l+D-2)\right]^{-1} r^{k+2} h_{l, m}(\hat{x}) .
\end{aligned}
$$

We remark that the order of the residue integrals is not arbitrary here; the integral over $\delta_{j+1}$ has to be performed first.

The above formula is not the only possible choice for $G$. In fact, any other choice $G^{\prime}$ of the right inverse compatible with $\Delta G=i d_{\mathcal{E}_{i}}, G: \mathcal{E}_{i} \rightarrow \mathcal{E}_{i+1}$ can be parametrized by constants $A_{j, l, m}, B_{j, l, m}, j, l \in \mathbb{N}, m \in\{1, \ldots, N(l, D)\}:$

$$
\begin{aligned}
G^{\prime}\left[r^{k} \ln ^{j} r h_{l, m}(\hat{x})\right]= & G\left[r^{k} \ln ^{j} r h_{l, m}(\hat{x})\right]+ \\
& \delta_{l}^{k+2} A_{j, l, m} r^{l} h_{l, m}(\hat{x})+\delta_{-l-D+2}^{k+2} B_{j, l, m} r^{-l-D+2} h_{l, m}(\hat{x}),
\end{aligned}
$$


where $\delta_{b}^{a}$ is the Kronecker delta. At each iteration step, we are free to choose one set of constants $A_{j, l, m}, B_{j, l, m}$.

This freedom is partly restricted by the consistency condition. One expects that the remaining freedom corresponds to the renormalization ambiguities in the conventional framework. As already said, we will not prove here that our particular choice for $G$ in eq. (4.46) actually leads to a set of vertex operators that fulfill the consistency condition.

\subsection{Graphical rules for computing vertex operators}

We now want to consider in more detail the iterative scheme for calculating vertex operators in perturbation theory described at the beginning of this section, based on our recipe for inverting $\Delta$ that we have just described.

\subsubsection{The case $D=2$ :}

We will explain this first for the OPE vertex algebra in $D=2$, with interaction $\lambda P(\varphi)=$ $\lambda \sum \frac{c_{p}}{p !} \varphi^{p}$. What makes the construction in this theory simple is that - as we will see - the "counterterms" in eq. (4.41) have no effect. This is directly related to fact that the field theory is "super-renormalizable" in field theory parlance. As usual in perturbation theory, it is of no concern that the interaction polynomial is not semi-bounded, even though this would render impossible the non-perturbative construction of the Schwinger functions.

In $D=2$, the angular part and the $N(l, D)=2$ harmonic polynomials at order $l>0$ are

$$
\hat{x}=\mathrm{e}^{\mathrm{i} \alpha}, \quad h_{l, \pm}(\hat{x})=\frac{1}{\sqrt{2 \pi}} \mathrm{e}^{ \pm \mathrm{i} l \alpha}
$$

At zeroth order, the vertex operator linear in $\varphi$ is given by

$$
Y_{0}(\varphi, x)=\mathbf{b}_{0} \ln r+\mathbf{b}_{0}^{+}+\sum_{l=1}^{\infty} \sum_{m= \pm 1} \frac{1}{\sqrt{2 l}}\left[r^{l} \mathrm{e}^{\mathrm{i} m l \alpha} \mathbf{b}_{l, m}^{+}+r^{-l} \mathrm{e}^{-\mathrm{i} m l \alpha} \mathbf{b}_{l, m}\right] .
$$

When determining the vertex operator $Y_{i+1}(\varphi, x)$ at order $i+1$, we have to calculate $Y_{i}\left(\varphi^{2}, x\right)$, see eq. (4.41), then $Y_{i}\left(\varphi^{3}, x\right)$ and so on, and then apply the inverse $G$ of $\Delta$ as in eq. (4.39). Eq. (4.41) contains what we called "counterterms", which are the terms with the --sign in front. However, it is not difficult to derive that, in $D$ spacetime dimensions, and for a polynomial interaction, the scaling degree (i.e., the power of $r$ of the most singular term apart from log's) of an $i$-th order vertex operator is

$$
\operatorname{sd}\left\langle c\left|Y_{i}(a, x)\right| b\right\rangle \leq \operatorname{dim}(a)+\operatorname{dim}(b)-\operatorname{dim}(c)+i\left[D-\frac{D-2}{2} \operatorname{deg}(P)\right],
$$

where dim is a grading of $V$ by the engineering dimension of the fields and is defined for a field of the form (3.29) by

$$
\operatorname{dim}(a)=\sum_{l, m} a_{l, m}\left(l+\frac{D-2}{2}\right)
$$


i.e., each factor of $\varphi$ counts as having dimension $\frac{D-2}{2}$, and each derivative as 1 , which gives $l$ for the $l$-th order differential operator $\bar{h}_{l, m}(\partial)$. It follows that, in $D=2$ dimension with a polynomial interaction, the scaling degree of all the terms $Y_{j}(\varphi, x-y) \varphi$ is strictly positive for $j>0$. Hence, we may drop the counterterms in perform the limit $|x-y| \rightarrow 0$ just on the first term. Thus, in $D=2$ and $i>0,(4.41)$ reads

$$
Y_{i}\left(\varphi^{2}, x\right)=\sum_{j=0}^{i} Y_{j}(\varphi, x) Y_{j-i}(\varphi, x),
$$

and an analogous equation can easily be derived for the vertex operators $Y_{i}\left(\varphi^{p}, x\right)$. The only difference is that we now have $p$ terms on the right side and a corresponding $p-1$-fold summation. We may thus form $Y_{i}\left(P^{\prime}(\varphi), x\right)$, and hence, using the field equation (4.38), we get $Y_{i+1}(\varphi, x)$. Explicitly, this is $(i>0)$

$$
Y_{i+1}(\varphi, x)=\sum_{p} \frac{c_{p}}{p !} G \sum_{j_{1}+\ldots+j_{p}=i} Y_{j_{1}}(\varphi, x) \cdots Y_{j_{p}}(\varphi, x) .
$$

For $i=0$, the formula is instead [compare eq. (3.35)]

$$
Y_{1}(\varphi, x)=\sum_{p} \frac{c_{p}}{p !} G: Y_{0}(\varphi, x)^{p}: .
$$

Each term in the sum over $p$ has an obvious graphical representation by a vertex with $p$ incoming lines, see fig. 1 .
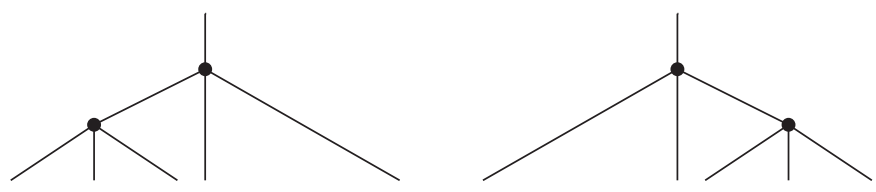

Figure 1: Two trees representing the terms $G\left(\left(G: Y_{0}(\varphi, x)^{3}:\right) Y_{0}(\varphi, x)^{2}\right)$ and $G\left(Y_{0}(\varphi, x)^{2}\left(G: Y_{0}(\varphi, x)^{3}:\right)\right)$ respectively. Even though the trees are related by a reflection, they have to be counted as different. Both make a contribution to the vertex operator $Y_{2}(\varphi, x)$ in the theory with interaction $\lambda P(\varphi)=\lambda \varphi^{4} / 4$ !.

Adopting such a graphical notation immediately helps one to see that if we iterate eq. (4.53) starting from $i=0$ to arbitrary $i$, then the resulting expression will be organized in terms of trees whose vertices have coordination number $p=1,2, \ldots, \operatorname{deg}(P)$, where $P$ was the polynomial characterizing the interaction. We use the notation $v \prec w$ to indicate 
that a vertex or leaf $v$ can be reached from the vertex $w$ following the tree downwards. By $v \preceq w$, we mean $(v \prec w$ or $v=w)$. As we have to heed the order of the creation and annihilation operators in the term represented by a tree, we have to count as different trees that are related to each other by a reflection or similar symmetry operation, see again fig. 1.

In the remainder of the subsection, we want to describe in somewhat more detail what the mathematical expression is for each such tree. Let $T$ be a tree on $i$ vertices, and let $Y_{i}(T, \varphi, x)$ be the contribution to $Y_{i}(\varphi, x)$ coming from that tree. Thus,

$$
Y_{i}(\varphi, x)=\sum_{\operatorname{trees} T \text { on } 1, \ldots, i} \frac{\prod c_{p_{v}}}{\prod p_{v} !} Y_{i}(T, \varphi, x),
$$

where $p_{v}$ is the coordination number of the vertex $v$. If we start from the leaves of the tree, then to each leaf $j$ ending in a vertex $v$ we have to associate a pair $\left(l_{j}, m_{j}\right) \in \mathbb{Z}_{+} \times\{ \pm 1\}$ and one of the following factors

$$
\frac{1}{\sqrt{2 l_{j}}} r^{l_{j}} \mathrm{e}^{\mathrm{i} m_{j} l_{j} \alpha} \mathbf{b}_{l_{j}, m_{j}}^{+} \quad \text { or } \quad \frac{1}{\sqrt{2 l_{j}}} r^{-l_{j}} \mathrm{e}^{-\mathrm{i} m_{j} l_{j} \alpha} \mathbf{b}_{l_{j}, m_{j}} .
$$

Or, if $l_{j}=0$, we have to associate one of the factors $\mathbf{b}_{0}^{+}$or $\oint \frac{\mathrm{d} \delta_{j}}{\delta_{j}} r^{\delta_{j}} \mathbf{b}_{0}$, using the residue trick to generate the logarithm. The creation/annihilation operators of the leaves associated with the same vertex must be normal ordered, by eq. (4.54). It is handy to distinguish the two cases in eq. (4.56) by calling the lines associated with the first type of factor "incoming", while calling "outgoing" the other one. For each tree, we will have to sum over all possible orientations of the leaves, because this will correspond to different terms contributing to $Y_{i}(\varphi, x)$.

Let us now consider a vertex $v$ that has no further vertices attached to it downwards in the tree; i.e. if we follow a line downwards starting from $v$, we arrive at a leaf. At $v$, we have to multiply the factors in eq. (4.56) associated to the leaves attached to $v$, put this product into normal order and then apply the inverse Laplacian $G$. It is efficient to take care of the phase factors by introducing, for each vertex, an auxiliary integration variable $0 \leq \beta \leq 2 \pi$, and to use the trivial identity

$$
\begin{aligned}
& \prod_{j \text { outgoing }} \mathrm{e}^{\mathrm{i} m_{j} l_{j} \alpha} \prod_{j \text { incoming }} \mathrm{e}^{-\mathrm{i} m_{j} l_{j} \alpha} \\
= & 2 \sum_{l=1}^{\infty} \int_{0}^{2 \pi} \frac{\mathrm{d} \beta}{2 \pi} \cos [l(\alpha-\beta)] \prod_{j \text { outgoing }} \mathrm{e}^{\mathrm{i} m_{j} l_{j} \beta} \prod_{j \text { incoming }} \mathrm{e}^{-\mathrm{i} m_{j} l_{j} \beta}+(l=0 \text { term }),
\end{aligned}
$$

which follows immediately using that the functions $\frac{1}{\sqrt{2 \pi}} \mathrm{e}^{ \pm \mathrm{i} l \alpha}$ form an orthonormal basis on $[0,2 \pi]$. The inverse of the Laplace operator has to be applied to an expression of the form $r^{\tilde{\nu}} \cos (l(\alpha-\beta))$, where $r^{\tilde{\nu}}$ results from collecting the powers of $r$ of the factors (4.56) associated with the leaves of $v$. The power is thus

$$
\tilde{\nu}=\sum_{j \text { outgoing }} l_{j}-\sum_{j \text { incoming }} l_{j},
$$


where we have assumed that none of the $l_{j}$ 's is zero. Now we introduce $\delta \in \mathbb{C} \backslash \mathbb{Z}$ and apply our residue trick from section 4.1 to evaluate $G\left(r^{\tilde{\nu}} \cos (l(\alpha-\beta))\right)$. (For each incoming line with $l_{j}=0$, we must replace the corresponding term with another such $\delta_{j}$.) The result is a contour integral with integrand $2 \frac{\cos (l(\alpha-\beta))}{\nu^{2}-l^{2}} r^{\nu+2}$, with $\nu=\tilde{\nu}+\delta$. (This holds for $l \neq 0$, for $l=0$ we have $\frac{1}{\nu^{2}} r^{\nu+2}$.) Having introduced the additional integration parameter $\beta$ now pays off, as we can carry out the sum over $l$ using the following formula for $\nu \in \mathbb{C} \backslash \mathbb{Z}$ :

$$
\frac{1}{2 \nu^{2}}+\sum_{l=1}^{\infty} \frac{\cos (l \alpha)}{\nu^{2}-l^{2}}=\frac{\pi}{\sin (\nu \pi)} \frac{\cos (\nu \alpha-\nu \pi)}{2 \nu}
$$

We can repeat this procedure for each of the remaining vertices of the tree, moving upwards in the tree. At each new vertex $v$ we introduce a new integration variable $\beta_{v}$, and a new summation variable $l_{v}$ - which is summed over using the above cosine identity - as well as a variable $\nu_{v}$ defined similarly as above. More precisely, when we use the residue trick to perform the inverse of the Laplace operator, we must first introduce for each vertex $v$ in the tree a new variable $\delta_{v} \in \mathbb{C} \backslash \mathbb{Z}$, and the residue in this variable then has to be taken in the end. If we do all this, we hence arrive at the following graphical rules for calculating $Y_{i}(T, \varphi, x)$, and hence $Y_{i}(\varphi, x)$ :

1. Draw the tree $T$ with $i$ vertices. Label the vertices by an index, $v$, and the lines by pairs of indices $(v w)$. The vertex $v$ has coordination number $p_{v}$. The leaves also carry indices.

2. With each leaf $j$ adjacent to a vertex $v$, associate a pair $\left(l_{j}, m_{j}\right) \in \mathbb{Z}_{+} \times\{ \pm 1\}$ and one of the following factors

$$
\frac{1}{\sqrt{2 l_{j}}} \mathrm{e}^{\mathrm{i} m_{j} l_{j} \beta_{v}} \mathbf{b}_{l_{j}, m_{j}}^{+} \quad \text { or } \quad \frac{1}{\sqrt{2 l_{j}}} \mathrm{e}^{-\mathrm{i} m_{j} l_{j} \beta_{v}} \mathbf{b}_{l_{j}, m_{j}} .
$$

The first factor is chosen if the line associated with the leaf is oriented upwards, and the second if it is oriented downwards. For the zero modes $\left(l_{j}=0\right)$, we have to associate one of the factors $\mathbf{b}_{0}^{+}$or $\oint \frac{\mathrm{d} \delta_{j}}{\delta_{j}} r^{\delta_{j}} \mathbf{b}_{0}$, where $\delta_{j} \in \mathbb{C} \backslash \mathbb{Z}$, again depending on the orientation. The creation/annihilation operators of the leaves connected to the same vertex are to be normal ordered.

3. With each vertex $v$ associate a parameter $\delta_{v} \in \mathbb{C} \backslash \mathbb{Z}$, and a parameter $\beta_{v} \in[0,2 \pi]$.

\footnotetext{
${ }^{4}$ This may be viewed as a degenerate case of the Dougall identity, see appendix A. To prove the identity, consider the contour integral

$$
\oint_{C} \frac{\cos (z(\alpha-\pi)) \mathrm{d} z}{z(\nu-z) \sin \pi z}
$$

where $C$ is a circle around the origin with radius $\pi(M+1 / 2), M \in \mathbb{N}$. For $M \rightarrow \infty$, the integral vanishes and we obtain eq. (4.59) via the residue theorem.
} 
4. With each vertex $v$ we associate $\nu_{v} \in \mathbb{C} \backslash \mathbb{Z}$ defined by

$$
\nu_{v}=\sum_{\text {in leaves } j \prec v} l_{j}-\sum_{\text {out leaves } j \prec v} l_{j}+\sum_{\text {vertices } w \preceq v}\left(2+\delta_{w}\right) .
$$

The "2" results from the inversion of the Laplacian, which at each inversion step (i.e., each vertex) raises the power of the radial coordinate by 2 . The $\delta_{w}$ arises from the residue trick for the Laplace inversion at each vertex $w$ below $v$.

5. With the root, associate the parameter $\alpha \in[0,2 \pi]$, and the factor $r^{\nu_{\text {root }}}$ collecting all the factors of $r$, where $x=r \mathrm{e}^{\mathrm{i} \text { alpha }}$. The number $\nu_{\text {root }} \in \mathbb{C}$ is defined as in eq. (4.61), but with the vertex $v$ replaced by the root, so that the sums contain contributions from all leaves of $T$.

6. With each line $(v w)$ (not connecting a leaf) associate a factor

$$
\frac{\pi}{\sin \left(\pi \nu_{w}\right)} \frac{\cos \left[\left(\beta_{v}-\beta_{w}\right) \nu_{w}-\pi \nu_{w}\right]}{\nu_{w}} .
$$

This results from the application of the cosine identity (4.59).

7. Perform the sum over all $l_{j}, m_{j}$ and zero modes. Furthermore, perform the integrals

$$
\prod_{\text {vertices } v} \int_{0}^{2 \pi} \mathrm{d} \beta_{v} \text { and } \prod_{\text {vertices } v} \frac{1}{2 \pi \mathrm{i}} \oint_{C_{v}} \frac{\mathrm{d} \delta_{v}}{\delta_{v}} .
$$

Finally, take the sum over all possible orientations of the leaves.

The last step requires some further comment. Our first comment concerns the choice of the integration contours $C_{v}$ in the residue integral. They can be chosen as small circles around the origin with radii $\left|\delta_{v}\right|$ chosen so that for any subset $\mathcal{V}$ of the vertices in the tree, and for any values of the phases of the $\delta_{v}, v \in \mathcal{V}$, we have $\sum_{v \in \mathcal{V}} \delta_{v} \in \mathbb{C} \backslash \mathbb{Z}$. We need this in order for our residue trick to work; the exponents $\nu_{v}$ have to be non-integer. The second remark concerns the convergence of the multiple summation over the counters $l_{j}$. To illustrate the general point, we consider the example tree with a fixed orientation of the leaves given in fig. 2 .

We apply the above rules and obtain the following expression for fig. 2:

$$
\begin{aligned}
& \oint_{C_{1}} \oint_{C_{2}} \frac{\mathrm{d} \delta_{v_{1}}}{\delta_{v_{1}}} \frac{\mathrm{d} \delta_{v_{2}}}{\delta_{v_{2}}} \int_{0}^{2 \pi} \int_{0}^{2 \pi} \mathrm{d} \beta_{v_{1}} \mathrm{~d} \beta_{v_{2}} \sum_{l_{1}, l_{2}, l_{3}} \sum_{m_{1}, m_{2}, m_{3}} \\
\times & \frac{\pi \cos \left[\left(\beta_{1}-\beta_{2}-\pi\right) \nu_{2}\right]}{\nu_{2} \sin \pi \nu_{2}} \frac{\pi \cos \left[\left(\alpha-\beta_{1}-\pi\right) \nu_{1}\right]}{\nu_{1} \sin \pi \nu_{1}} \\
\times & e^{\mathrm{i} \text { beta }_{2}\left(-m_{1} l_{1}-m_{2} l_{2}\right)} e^{\mathrm{i} \text { beta }_{1} m_{3} l_{3}} r^{\nu_{1}} \mathbf{b}_{l_{1}, m_{1}}^{+} \mathbf{b}_{l_{2}, m_{2}}^{+} \mathbf{b}_{l_{3}, m_{3}}
\end{aligned}
$$




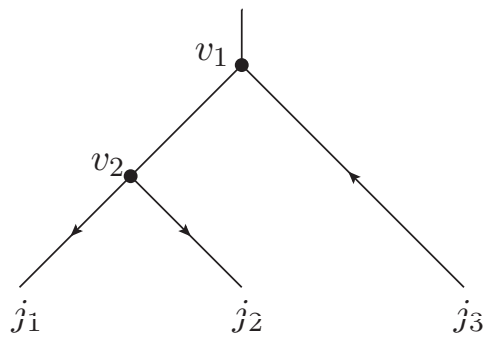

Figure 2: Tree contributing to $Y_{2}(\varphi, x)$, with fixed orientation of the leaves.

with $\nu_{2}=-l_{1}-l_{2}+2+\delta_{2}, \nu_{1}=-l_{1}-l_{2}+l_{3}+4+\delta_{1}+\delta_{2}$, which makes a contribution to the vertex operator $Y_{2}(\varphi, x)$ in the theory with interaction $\lambda P(\varphi)=\lambda \varphi^{3} / 3$ !. We are going to normal-order the product of creation and annihilation operators $\mathbf{b}_{l_{1}, m_{1}}^{+} \mathbf{b}_{l_{2}, m_{2}}^{+} \mathbf{b}_{l_{3}, m_{3}}$, which will make it easier to calculate matrix elements of the vertex operator. (Recall that the vertex operators were related to the OPE coefficients by the formula $\langle c|Y(a, x)| b\rangle=C_{a b}^{c}(x)$.) The only terms for which normal ordering has non-trivial effects are those where either $\left(l_{1}, m_{1}\right)=\left(l_{3}, m_{3}\right)$ or $\left(l_{2}, m_{2}\right)=\left(l_{3}, m_{3}\right)$. These terms can be visualized by joining the leaves $j_{1}, j_{3}$ or $j_{2}, j_{3}$ respectively by a new line, see fig. 3 .

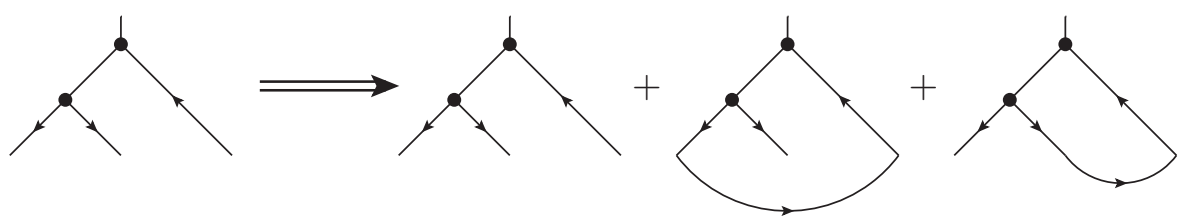

Figure 3: Application of Wick's theorem to a tree. On the left side of the arrow, we have the tree from fig. 2. On the right side, we have the representation of the normal ordered expression for it. Contractions are represented by an additional line.

We see that, in general, we will "close loops" in the tree when we apply Wick's theorem (see eq. (C.175)) to the products of creation and annihilation operators. Thus, when normal ordering the operators in the expression $Y_{i}(T, \varphi, x)$, we get contractions between the creation and annihilation operators between the individual leaves (not attached to the same vertex, as these already are normal ordered).

Let $j$ and $k$ be leaves. Let $j$ be outgoing and standing to the left of $k$, which shall be incoming. Their contraction gives $\delta_{l_{j}, l_{k}} \delta_{m_{j}, m_{k}}$. We combine this with the other factors in eq. (4.60) and carry out the sum over $m_{j}$, getting $\frac{\cos \left[l_{j}\left(\beta_{v}-\beta_{w}\right)\right]}{l_{j}}$, when $l_{j} \neq 0$, where $v$ is the vertex adjacent to $j$ and $w$ the vertex adjacent to $k$. When $l_{j}=0$, we get instead $\frac{1}{\delta_{j}}$. We will 
represent each such new factor by a new line joining the respective leaves. If we apply this systematically, we are thus led to a wider class of graphical objects that are obtained from our tree $T$ by joining an arbitrary number of leaves, but never joining two leaves from the same vertex. These new graphs, which we call $G$, are not trees any more, but also contain loops. The orientation of the loops is always from the "left" to the "right", pointing from a leaf representing a creation operator to a leaf representing an annihilation operator. In the language of graphs, $T$ is a "spanning tree" for each $G$, and we collect these graphs in the set

$$
\mathcal{G}(T)=\{\text { graphs } G \mid T \text { a spanning tree for } G\} .
$$

Our graphs $G$ contain three different kinds of lines, or edges $e$ : The edges $e \in T$ that were already present in the tree $T$, the edges $e \in G \backslash T$ that were created by joining two leaves, and the leaves $e$ that were not joined (the "external lines"). The edges in the first category carry momenta $\nu_{e} \in \mathbb{C}$ that are determined by the momentum conservation rule (4.61). The edges in the second category carry "loop" momentum $l_{e} \in \mathbb{Z}$, and the leaves e carry "external" quantum numbers $l_{e}, m_{e}$. The collection of loop and external momenta together is the same as the assignment $l_{e}$ above. We denote by $Y_{i}(G, \varphi, x)$ the contribution to $Y_{i}(\varphi, x)$ from such an individual loop graph $G$ where the sum over all possible orientations of the external lines is understood. In other words,

$$
Y_{i}(\varphi, x)=\sum_{\text {trees } T \text { on } 1, \ldots, i} \frac{\prod c_{p_{k}}}{\prod p_{k} !} \sum_{G \in \mathcal{G}(T)} Y_{i}(G, \varphi, x) .
$$

The contribution $Y_{i}(G, \varphi, x)$ from an individual graph $G \in \mathcal{G}(T)$, is given in turn by

$$
\begin{aligned}
Y_{i}(G, \varphi, x) & =\sum_{\substack{\text { leaf } \\
\text { orientations }}} \sum_{l_{e}, m_{e}, e \text { leaf }} \sum_{l_{e}, e \in G \backslash T}\left(\prod_{\text {vertices } v} \int_{C_{v}} \frac{\mathrm{d} \delta_{v}}{\delta_{v}} \int_{0}^{2 \pi} \mathrm{d} \beta_{v}\right) \\
& \times \prod_{e \in T} \frac{\pi}{\sin \pi\left(l_{e}+\delta_{e}\right)} \frac{\cos \left[\left(l_{e}+\delta_{e}\right)\left(\beta_{e}-\pi\right)\right]}{l_{e}+\delta_{e}} \prod_{e \in G \backslash T} \frac{\cos \left(\beta_{e} l_{e}\right)}{l_{e}} \\
& \times \exp \left(\ln r\left\{\sum_{\text {in leaves } e} l_{e}-\sum_{\text {out leaves } e} l_{e}+\sum_{\text {vertices } v}\left(2+\delta_{v}\right)\right\}\right) \\
& \times: \prod_{\text {leaves } e} \mathbf{b}_{l_{e}, m_{e}}^{ \pm} \frac{\mathrm{e}^{ \pm \mathrm{i} m_{e} l_{e} \beta_{e}}}{\sqrt{2 l_{e}}}: .
\end{aligned}
$$

This formula requires several comments. First, we have used now the notation " $e$ " for the edges of $e$, which include those present already in $T$, and those that close the loops, i.e. in $G \backslash T$. To each of the latter, we have associated an integer $l_{e} \in \mathbb{N}$, which is summed over. Each of the lines $e$ already present in $T$ also carries an $l_{e} \in \mathbb{Z}$, which is determined by the "conservation rule"

$$
2=\sum_{e \text { outgoing }} l_{e}-\sum_{e \text { incoming }} l_{e}
$$


at every vertex $v$, where the incoming lines $e$ are either incoming leaves or lines below $v$ (in the sense of the relation $\prec$ ), and where the outgoing lines $e$ are either outgoing leaves or the line above $v$ (in the sense of the relation $\succ$ ). Hence, the numbers $l_{e}, e \in T$ are determined by the $L$ loop momenta $l_{e}, e \in G \backslash T$ and external momenta (leaves) $l_{e}$ via the momentum conservation rule at the vertices of $G$. That momentum conservation rule comes from eq. (4.61). We have also introduced $\delta_{e}$ as the sum $\sum_{v} \delta_{v}$ of all those $\delta_{v} \in \mathbb{C} \backslash \mathbb{Z}$ that are associated with a vertex "below" the line $e$ in the original tree, and this accounts for the corresponding term in eq. (4.61). Finally, also the leaves (i.e. uncontracted lines) carry indices $l_{e}, m_{e}$ that are summed, and we have set $\beta_{e}=\beta_{v}-\beta_{w}$ if $e=(v w)$, with $\beta_{v}=\alpha$ if $v$ is the root, and $\beta_{e}=\beta_{v}$ if $e$ is a leaf associated to the vertex $v$. As always, $x$ is related to $r$ and $\alpha$ by $x=r \mathrm{e}^{\mathrm{i} \alpha}$.

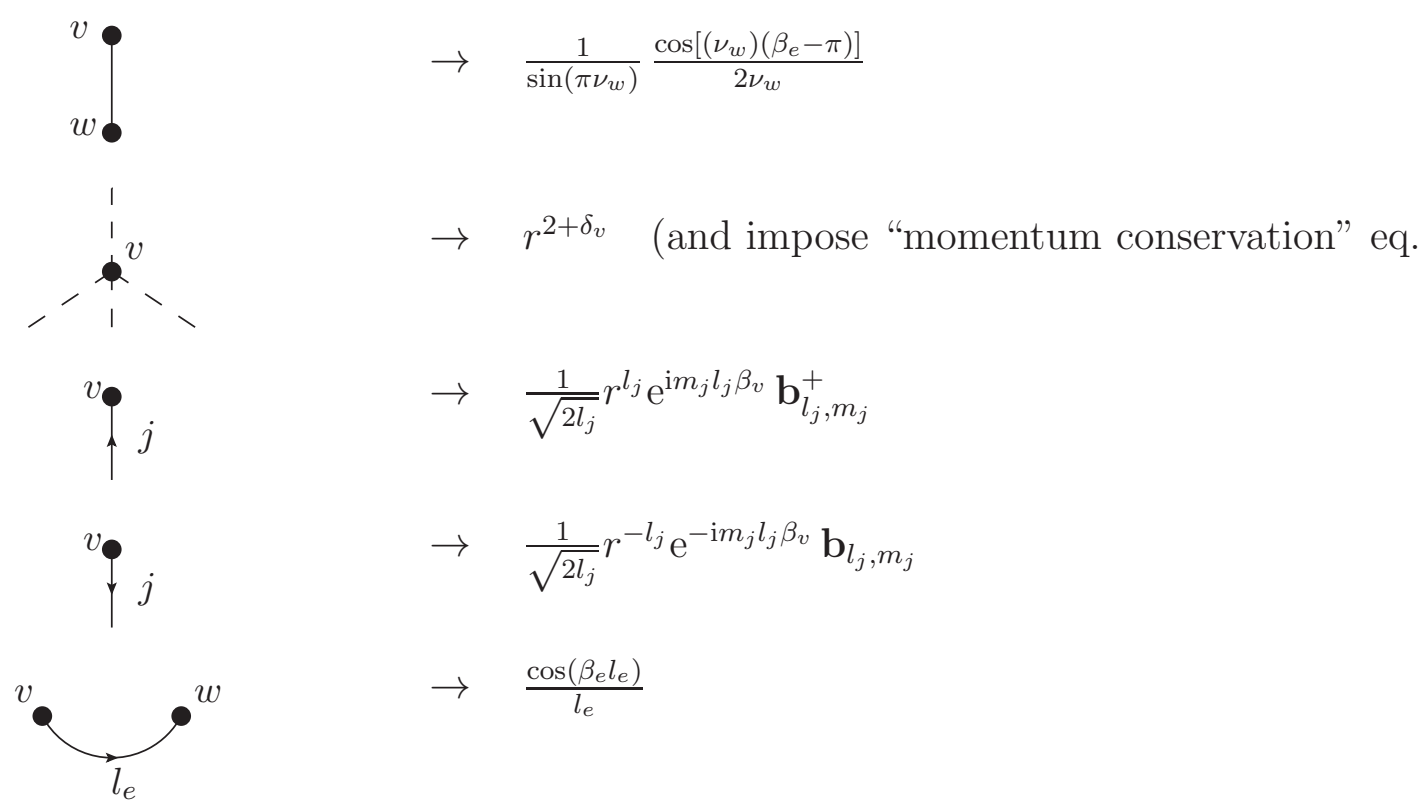

Figure 4: The rules for the "amplitude" of a graph. The appropriate summations and integrals have to be understood. Slightly deviating from the earlier statement of the rules, we do not associate all powers of $r$ with the root but all leaves and all vertices carry some power of $r$.

The formula for $Y_{i}(G, \varphi, x)$ given above looks quite complicated. To see that the sums converge, we now introduce the following trick that, in effect, replaces the sums by integrals. 
The basic trick is to implement the conservation rule (4.66) by the integral

$$
\int_{0}^{2 \pi} \frac{\mathrm{d} t_{v}}{2 \pi} \exp \left(\mathrm{i} t_{v}\left\{2+\sum_{e=(v w): v \succ w} l_{e}-\sum_{e=(v w): v \prec w} l_{e}\right\}\right)
$$

with one new integration variable $t_{v}$ per vertex $v$ because momentum conservation holds at each vertex. Once the momentum conservation rule has been implemented (at the expense of the new integrals), we can now sum over all $l_{e}$ independently, and not just the $l_{e}$ associated with the loop lines $e \in G \backslash T$. We then do the new integrals afterwards. The advantage is that the sums can now be performed explicitly. We hence trade the summations over $l_{e}, e \in G \backslash T$ for the integrals over the $t_{v}$, where $v$ runs through the vertices, as we now explain in more detail.

The infinite summations that we have to deal with occur at each line $e \in G$ that is not an external line (leaf), and they are performed with the aid of the formula

$$
\sum_{l=0}^{\infty} \frac{\cos [(l+\delta) \beta] h^{l}}{l+\delta}=\frac{1}{2 \delta}\left(\mathrm{e}_{2}^{\mathrm{i} \delta \beta} F_{1}\left(\delta, 1 ; 1+\delta ; h \mathrm{e}^{\mathrm{i} \beta}\right)+\mathrm{e}_{2}^{-\mathrm{i} \delta \beta}{ }_{2} F_{1}\left(\delta, 1 ; 1+\delta ; h \mathrm{e}^{-\mathrm{i} \beta}\right)\right)
$$

which holds for $\delta \in \mathbb{C} \backslash \mathbb{Z}$, and $|h|<1$, and which follows straightforwardly from the definition of the Gauss hypergeometric series, see appendix A. In the above complicated formula (4.65) for $Y_{i}(G, \varphi, x)$, we now apply the formula at each internal line $e \in T$, with the choice $l=l_{e}$, $\delta=\delta_{e}, \alpha=\beta_{e}$, and $h=\mathrm{e}^{\mathrm{i} t_{e}}$, where $t_{e}=t_{v}-t_{w}$ for the line $e=(v w)$. For the loop lines $(e \in G \backslash T)$, we have the formula

$$
\sum_{l \in \mathbb{N}} \frac{\cos (l \alpha)}{l} h^{l}=-\ln \sqrt{1+h^{2}-2 h \cos \alpha}
$$

However, we cannot apply these formulae straightforwardly, for two reasons. First, they only hold for $|h|<1$, while our choice would correspond to $|h|=1$, which is on the boundary of the disk of convergence of the Gauss hypergeometric series. Secondly, the series in $Y_{i}(G, \varphi, x)$ have to go over both positive and negative values of $l_{e}$ if $e$ is neither a leaf nor a loop line, whereas eq. (4.68) only goes over non-negative values. The remedy to this difficulty is as follows. We first split each sum over $l_{e} \in \mathbb{Z}$ into positive and negative values of $l_{e}$. Then, for the positive values of $l_{e}$, we replace $t_{e}$ by $t_{e}+\mathrm{i} 0$ (i.e., we add a small positive imaginary part), and for negative values of $l_{e}$ we replace $t_{e}$ by $t_{e}-\mathrm{i} 0$. This then justifies the exchange of summation and integration over the $t_{v}$ before we take the small imaginary part to zero, and the remaining integration over the $t_{v}$ must then be understood in the sense of distributions.

The trick affects our formula (4.65) for $Y_{i}(G, \varphi, x)$ as follows. Instead of the sum/integral in the first line of eq. (4.65), we now have

$$
\sum_{\substack{\text { leaf } \\ \text { orientations }}} \sum_{l_{e}, m_{e}, e \text { leaf }}\left(\prod_{\text {vertices } v} \int_{C_{v}} \frac{\mathrm{d} \delta_{v}}{\delta_{v}} \int_{0}^{2 \pi} \mathrm{e}^{2 \mathrm{i} t_{v}} \mathrm{~d} t_{v} \int_{0}^{2 \pi} \mathrm{d} \beta_{v}\right)
$$


i.e., we have gotten rid of the - potentially dangerous - summation over the loop momenta, and replaced these by additional integrations, which are easier to control as we will see in a moment. Furthermore, the term in the first product $(e \in T)$ in the second line becomes

$$
\begin{aligned}
g_{2}\left(\delta_{e}, \cos \beta_{e}, t_{e}\right):=\frac{\pi}{\sin \pi \delta_{e}} & \left(\sum_{l=0}^{\infty}(-1)^{l} \mathrm{e}^{\mathrm{i}\left(t_{e}+10\right) l} \frac{\cos \left[\left(l+\delta_{e}\right) \beta_{e}\right]}{l+\delta_{e}}\right. \\
& \left.+\sum_{l=-\infty}^{-1}(-1)^{l} \mathrm{e}^{\mathrm{i}\left(t_{e}-10\right) l} \frac{\cos \left[\left(l+\delta_{e}\right) \beta_{e}\right]}{l+\delta_{e}}\right) .
\end{aligned}
$$

Using eq. (4.68), we get

$$
\begin{aligned}
& g_{2}\left(\delta_{e}, \cos \beta_{e}, t_{e}\right)= \\
& \frac{\mathrm{e}^{+\mathrm{i} \delta_{e} \beta_{e}}}{2 \delta_{e} \sin \pi \delta_{e}}\left({ }_{2} F_{1}\left(\delta_{e}, 1 ; 1+\delta_{e} ;-\mathrm{e}^{\mathrm{i}\left(+\beta_{e}+t_{e}+\mathrm{i} 0\right)}\right)-{ }_{2} F_{1}\left(-\delta_{e}, 1 ; 1-\delta_{e} ;-\mathrm{e}^{\mathrm{i}\left(-\beta_{e}-t_{e}+\mathrm{i} 0\right)}\right)-1\right) \\
+ & \frac{\mathrm{e}^{-\mathrm{i} \delta_{e} \beta_{e}}}{2 \delta_{e} \sin \pi \delta_{e}}\left({ }_{2} F_{1}\left(\delta_{e}, 1 ; 1+\delta_{e} ;-\mathrm{e}^{\mathrm{i}\left(-\beta_{e}+t_{e}+\mathrm{i} 0\right)}\right)-{ }_{2} F_{1}\left(-\delta_{e}, 1 ; 1-\delta_{e} ;-\mathrm{e}^{\mathrm{i}\left(+\beta_{e}-t_{e}+\mathrm{i} 0\right)}\right)-1\right) .
\end{aligned}
$$

The term in the second product $(e \in G \backslash T)$ is as in eq. (4.69). The remaining parts of the formula (4.65) are unchanged. The total effect of these manipulations is reflected in eq. (5.114), setting $D=2$ and $\epsilon_{v}=0$ there.

A possible divergence can now only come from the $\mathrm{d} \beta_{v} \mathrm{~d} t_{v}$ integrations, and the danger can only come from configurations near $\beta_{e}= \pm t_{e}+\pi$, where the argument of the hypergeometric function tends to 1 . It is at this stage that having integrals instead of sums pays off, because we can now use the well-known expansion formula for the Gauss hypergeometric function near $x=1$ :

$$
{ }_{2} F_{1}(\delta, 1 ; 1+\delta ; x)=2 \delta\left[\gamma_{\mathrm{E}}-\psi(\delta)\right]-1-\delta \ln (1-x)+o(1-x) .
$$

When we apply this to the ${ }_{2} F_{1}$-factors in the terms above, we find that

$$
g_{2}\left(\delta_{e}, \cos \beta_{e}, t_{e}\right) \sim-\frac{\mathrm{e}^{\mathrm{i} \delta_{e} \beta_{e}}}{2 \sin \left(\delta_{e} \beta_{e}\right)} \ln \left|\beta_{e}-t_{e}-\pi\right|+\frac{\mathrm{e}^{-\mathrm{i} \delta_{e} \beta_{e}}}{2 \sin \left(\delta_{e} \beta_{e}\right)} \ln \left|\beta_{e}+t_{e}-\pi\right|,
$$

near $\beta_{e}= \pm t_{e}+\pi$, where we have also used standard identities such as $\ln (x+\mathrm{i} 0)=\ln |x|+$ $\mathrm{i} \pi \theta(x)$. The contour integrals over the parameters $\delta_{e}$ are harmless as long as we choose the contours such that $\operatorname{dist}\left(\delta_{e}, \mathbb{Z}\right)>$ const. These estimates suffice to show that any matrix element $\left\langle c\left|Y_{i}(\varphi, x)\right| b\right\rangle$ is convergent. To see this, we recall that $Y_{i}(\varphi, x)$ was the sum over all trees $T$ on $i$ elements of the quantities $Y_{i}(T, \varphi, x)$, which in turn was the sum over all $G \in \mathcal{G}(T)$ of the quantities $Y_{i}(G, \varphi, x)$, for which we gave a formula above. Now, when forming a matrix element $\left\langle c\left|Y_{i}(G, \varphi, x)\right| b\right\rangle$, we get a sum of terms of the form

$$
\left\langle c\left|: \prod_{\text {leaves } e} \mathbf{b}_{l_{e}, m_{e}}^{ \pm} \frac{\mathrm{e}^{ \pm \mathrm{i} m_{e} l_{e} \beta_{e}}}{\sqrt{2 l_{e}}}:\right| b\right\rangle .
$$


We write $\langle c|=(c !)^{-1 / 2}\langle 0| \prod\left(\mathbf{b}_{l, m}\right)^{c_{l, m}}$ and $|b\rangle=(b !)^{-1 / 2} \prod\left(\mathbf{b}_{l, m}^{+}\right)^{b_{l, m}}|0\rangle$. Furthermore, for a fixed assignment of orientations and indices $l_{e}, m_{e}$ to each of the (uncontracted) leaves $e \in G$, we introduce the multiindices $a^{+}, a^{-}$by

$$
\begin{aligned}
& a_{l, m}^{+}=\#\left\{e \in G: e \text { incoming },\left(l_{e}, m_{e}\right)=(l, m)\right\} \\
& a_{l, m}^{-}=\#\left\{e \in G: \text { e outgoing, }\left(l_{e}, m_{e}\right)=(l, m)\right\} .
\end{aligned}
$$

In order for the matrix element (4.75) not to vanish, all creation operators have to be contracted with annihilation operators of the same indices. Remember that we are considering the contribution of a graph $G$ with a fixed number of leaves. In the above notation, that means $\left|a^{+}\right|+\left|a^{-}\right|$is fixed. We also assume that the multiindices $b, c$ are fixed. This means there is maximally one choice for $a^{+}, a^{-}$so that the matrix element (4.75) does not vanish. In this case the latter is equal to

$$
(4.75)=\prod_{e \text { incoming }} \frac{\mathrm{e}^{\mathrm{i} m_{e} l_{e} \beta_{e}}}{\sqrt{2 l_{e}}} \prod_{e \text { outgoing }} \frac{\mathrm{e}^{-\mathrm{i} m_{e} l_{e} \beta_{e}}}{\sqrt{2 l_{e}}} .
$$

The sum over the orientations and indices of the leaves in eq. (4.65) thus reduces to the sum over all graphs with $\left|a^{+}\right|$incoming leaves carrying indices from $a^{+}$and $\left|a^{-}\right|$outgoing leaves carrying indices from $a^{-}$. This sum is obviously finite.

Thus, the only source of divergent behavior are the integrals over the $\beta_{v}, t_{v}$. By our estimates above, these are bounded from above by (a constant times) the integral of the form

$$
I_{G}=\prod_{\operatorname{vertices} v \text { in } G}\left(\int_{0}^{2 \pi} \mathrm{d} \beta_{v} \int_{0}^{2 \pi} \mathrm{d} t_{v}\right) \prod_{e \in G} \ln \left|t_{e} \pm \beta_{e}-\pi\right| .
$$

Such integrals are standard "loop integrals" in quantum field theory (in position space), and they are well-known to be finite. Hence we conclude that our formula for $Y_{i}(\varphi, x)$ gives a finite answer, despite the infinite sums.

\subsubsection{The case $D>2$}

Let us now consider the field theory with interaction $\lambda P(\varphi)=\lambda \frac{1}{4 !} \varphi^{4}$, in dimension $D>2$. The main difference to $D=2$ is that eq. (4.52), and hence eq. (4.53), no longer holds, as the "counterterms" in eq. (4.41) now do not have a vanishing limit as $|x-y| \rightarrow 0$. This is closely related to the fact that, for $D=2$, we were dealing with the special case of a super-renormalizable theory whose divergences are of a particularly benevolent nature. In dimensions $D>2$, we must work with a version of eq. (4.41) that also incorporates the counterterms, and this will lead to correspondingly more complicated graphical rules. 
Our aim is to express the vertex operator $Y_{i}(\varphi, x)$ as a function of the already known 0 -th order vertex operators, and then find a graphical representation for this expression. We list the equations that we need to decompose $Y_{i}(\varphi, x)$ into 0-th order operators. Eq. (4.39) reads

$$
Y_{i+1}(\varphi, y)=\frac{1}{3 !} G Y_{i}\left(\varphi^{3}, y\right)
$$

Moreover, we need the equations (4.41) and

$$
Y_{i}\left(\varphi^{3}, y\right)=\sum_{j=0}^{i} Y_{j}(\varphi, x) Y_{i-j}\left(\varphi^{2}, y\right)-\sum_{j=1}^{i} Y_{i-j}\left(Y_{j}(\varphi, x-y) \varphi^{2}, y\right)-\frac{1}{|x-y|^{D-2}} Y_{i}(\varphi, y)+\ldots
$$

where the dots stand again for terms vanishing in the limit $x-y \rightarrow 0$.

We do not perform the limit $x-y \rightarrow 0$ for the moment, so each time we use either of the eqs. (4.41) or (4.80), we have to introduce a new variable from $\mathbb{R}^{D}$. We can choose this new variable $(x$ above) to be $(1+\epsilon)$ times the old variable $(y$ above, i.e. $x=(1+\epsilon) y)$, where a new regulator $\epsilon_{v}>0$ has to be introduced each time we apply either of eqs. (4.41) or (4.80). The result of repeatedly applying eqs. (4.79), (4.41) and (4.80) is a sum of products of nested 0 -th order vertex operators whose arguments from $\mathbb{R}^{D}$ depend on the initial variable from $\mathbb{R}^{D}$ and the $\epsilon_{v}$ 's. We now focus on a special partial sum, the sum of "tree-like" summands. We call a summand tree-like if, when tracing back its path through the iteration process, at each iteration step it does neither belong to the counterterms nor to the smooth terms represented by dots. Another way to put this is to say that we are only interested in the terms that we would have obtained by dropping the counterterms and dots in eqs. (4.41) and (4.80).

A formal definition of the tree-like summands can be given as follows. Let $T$ be a tree such that all vertices have coordination number four. With each vertex or leaf $v$ of the tree associate a regulator $\epsilon_{v}$. If we have three such trees $T_{1}, T_{2}, T_{3}$ with number vertices $i_{1}, i_{2}, i_{3}$, then we can join these at their root to form a new tree, $T=\cup_{w} T_{w}$ with $i$ vertices. The three vertices now attached to the new root (corresponding to the 3 subtrees $T_{1}, T_{2}, T_{3}$ ) carry new regulators denoted e.g. $\epsilon_{1}, \epsilon_{2}, \epsilon_{3}$. The recursive definition of the tree-like contributions to the $i$-th order vertex operators is then

$$
Y_{i}(T, \varphi, x)=\frac{1}{3 !} \sum_{\substack{i_{1}+i_{2}+i_{3}=i \\ \cup_{w} T_{w}=T}} \prod_{w=1}^{3} Y_{i_{w}}\left(T_{w}, \varphi, x_{w}\right)
$$

where $x_{w}=\left(1+\epsilon_{w}\right) x$, and where the dependency of the quantities $Y_{i}(T, \varphi, x)$ etc. on the regulators $\left\{\epsilon_{v}\right\}$ has been suppressed to lighten the notation. The vertex operators $Y_{i}(\varphi, x)$ are given by a sum of these tree-like terms [see eq. (4.55)], plus counterterms. Moreover, it will turn out that these tree-like summands can be thought of as building blocks for the (more complicated) counterterms. We will not discuss these here, but 
outline their construction in the next section. The regulators $\left\{\epsilon_{v}\right\}$ cannot be sent to zero before taking the sum of all contributions to the vertex operator, including the counterterms.

We would now like to find a closed form expression for the tree-like terms, applying the same kind of reasoning as for $D=2$. We have to take into account some differences. The first difference is that the trigonometric functions in the expression for the free vertex operator are now replaced by the harmonic polynomials, see eq. (3.28). The analogue of relation $(4.57)$ is

$$
\begin{aligned}
& \prod_{j \text { outgoing }} h_{l_{j}, m_{j}}(\hat{x}) \prod_{j \text { incoming }} \bar{h}_{l_{j}, m_{j}}(\hat{x}) \\
= & \sum_{l=0}^{\infty} \frac{2 l+D-2}{\sigma_{D}} \int_{S^{D-1}} \mathrm{~d} \Omega(\hat{y}) \mathrm{P}(D, l, \hat{x} \cdot \hat{y}) \prod_{j \text { outgoing }} h_{l_{j}, m_{j}}(\hat{y}) \prod_{j \text { incoming }} \bar{h}_{l_{j}, m_{j}}(\hat{y}),
\end{aligned}
$$

using this time the orthogonality of the harmonic polynomials, as well as the formula for the Legendre polynomials $\mathrm{P}(z, l, D)$ in $D$ dimensions given in appendix A. We proceed as in the case of $D=2$ dimensions. Let us now consider a vertex $v$ that has no further vertices attached to it downwards in the tree. Let the leaves attached to $v$ be labeled by $j$. We collect corresponding factors of $r$, and the harmonic polynomials. The harmonic polynomials are multiplied by the formula just given, while the factors of $r$ work out as $r^{\tilde{\nu}_{v}}$, where $\tilde{\nu}_{v}$ is now given by

$$
\tilde{\nu}_{v}=\sum_{j \text { incoming }} l_{j}-\sum_{j \text { outgoing }}\left(l_{j}+D-2\right) .
$$

Thus, in total we get a factor of $(2 l+D-2) r^{\tilde{\nu_{v}}} \mathrm{P}(\hat{x} \cdot \hat{y}, l, D)$. We have to apply the inverse of the Laplacian to this expression using the residue trick, introducing $\delta_{v}$ and $\nu_{v}=\tilde{\nu}_{v}+\delta_{v}$. When we do this, we get a contour integral with integrand

$$
\frac{(2 l+D-2) \mathrm{P}(\hat{x} \cdot \hat{y}, l, D) r^{\nu+2}}{\nu_{v}\left(\nu_{v}+D-2\right)-l(l+D-2)} .
$$

The sum over $l$ is now readily performed ${ }^{5}$ using the generalized Dougall's identity, see appendix $\mathrm{B}$, which states that for any $\nu \in \mathbb{C} \backslash \mathbb{Z}$ and $-1 \leq z \leq+1$ and $D \geq 3$, we have the identity

$$
\sum_{l=0}^{\infty} \frac{(2 l+D-2) \mathrm{P}(z ; l, D)}{\nu(\nu+D-2)-l(l+D-2)}=\frac{\pi}{\sin \pi \nu} \mathrm{P}(-z, \nu, D) .
$$

From the vertices connected to the leaves, we then work our way upwards, repeating for each new vertex the same procedure. We will then end up with a similar set of graphical rules. The main difference to $D=2$ is that we have to use Legendre functions instead of the

\footnotetext{
${ }^{5}$ This key observation is due to [13].
} 
trigonometric functions, and that we must take into account the regulators $\epsilon_{v}$. As one can see, this means that we have to introduce an extra factor

$$
\begin{aligned}
&\left(\left(1+\epsilon_{j}\right) \prod_{\text {vertices } v \succeq j}\left(1+\epsilon_{v}\right)\right)^{l_{j}} \\
& \text { or } \quad\left(\left(1+\epsilon_{j}\right) \prod_{\text {vertices } v \succeq j}\left(1+\epsilon_{v}\right)\right)^{-l_{j}-D+2}
\end{aligned}
$$

for each leaf $j$, depending on whether it is incoming or outgoing, to get the correct rules for $D>2$.

To summarize, we have the following graphical rules for calculating $Y_{i}(T, \varphi, x)$, the regularized contribution of a tree $T$ to the vertex operator $Y_{i}(\varphi, x)$ for the theory with interaction $\lambda P(\varphi)=\lambda \varphi^{4} / 4$ ! in $D>2$ (a general interaction polynomial is completely analogous):

1') Draw all trees with $i$ vertices with incidence number 4 . Label the vertices by an index $v$ and the lines by pairs of indices $(v w)$. The leaves also carry indices.

2') With each vertex $v$ associate a parameter $\delta_{v} \in \mathbb{C} \backslash \mathbb{Z}$, a parameter $\hat{y}_{v} \in S^{D-1}$ and a regulator $\epsilon_{v}>0$.

3') With each leaf $j$ adjacent to a vertex $v$, associate a pair $\left(l_{j}, m\right) \in \mathbb{N} \times\{1, \ldots, N(D, l)\}$ and one of the following factors

$$
\begin{aligned}
& \frac{K_{D}}{\sqrt{\omega\left(D, l_{j}\right)}} h_{l_{j}, m_{j}}\left(\hat{y}_{v}\right) \mathbf{b}_{l_{j}, m_{j}}^{+} \\
\text {or } \quad & \frac{K_{D}}{\sqrt{\omega\left(D, l_{j}\right)}} \overline{h_{l_{j}, m_{j}}}\left(\hat{y}_{v}\right) \mathbf{b}_{l_{j}, m_{j}}
\end{aligned}
$$

The first factor is chosen if the line associated with the leaf is oriented upwards, and the second if it is oriented downwards. The creation/annihilation operators of the leaves connected to the same vertex are to be normal ordered. Also, write down the regularizing factor

$$
\begin{gathered}
\left(\left(1+\epsilon_{j}\right) \prod_{\text {vertices } v \succeq j}\left(1+\epsilon_{v}\right)\right)^{l_{j}} \\
\text { or } \quad\left(\left(1+\epsilon_{j}\right) \prod_{\text {vertices } v \succeq j}\left(1+\epsilon_{v}\right)\right)^{-l_{j}-D+2}
\end{gathered}
$$

4') With each vertex $v$ we associate $\nu_{v} \in \mathbb{C} \backslash \mathbb{Z}$ defined by

$$
\nu_{v}=\sum_{\text {in leaves } j \prec v} l_{j}-\sum_{\text {out leaves } j \prec v}\left(l_{j}+D-2\right)+\sum_{\text {vertices } w \preceq v}\left(2+\delta_{w}\right) .
$$

The "2" results from the inversion of the Laplacian, which at each inversion step (i.e., each vertex) raises the power of the radial coordinate by 2 . The $\delta_{w}$ arises from the residue trick for the Laplace inversion at each vertex $w$ below $v$. 
5') With the root, associate the parameter $\hat{x} \in S^{D-1}$, and the factor $r^{\nu_{\text {root }}}$, where $x=r \hat{x}$. The number $\nu_{\text {root }} \in \mathbb{C}$ is defined as in eq. (4.61), but with the vertex $v$ replaced by the root, so that the sums contain contributions from all leaves of $T$.

6') With each line $(v w)$ connecting vertices $v, w$ associate a factor

$$
\frac{\pi}{\sin \pi \nu_{w}} \mathrm{P}\left(-\hat{y}_{v} \cdot \hat{y}_{w} ; \nu_{w}, D\right)
$$

This results from the application of the Dougall formula.

7') Perform the sum over all $l_{j}, m_{j}$. Furthermore, perform the integrals

$$
\prod_{\text {vertices } v} \int_{S^{D-1}} \mathrm{~d} \Omega\left(\hat{y}_{v}\right) \text { and } \prod_{\text {vertices } v} \frac{1}{2 \pi \mathrm{i}} \oint_{C_{v}} \frac{\mathrm{d} \delta_{v}}{\delta_{v}}
$$

Finally, take the sum over all possible orientations of the leaves.

If we proceed as in the case $D=2$ and perform the sum of all tree-like terms as in eqs. (4.64), (4.55) we do not get the complete vertex operator $Y_{i}(\varphi, x)$, so in this sense our rules are not complete. To get the complete vertex operator, we should also incorporate the counterterms in $D>2$ (see next section). The sum over graphs (4.64) depends on the regulators $\epsilon_{v}$, and is divergent for $\epsilon_{v} \rightarrow 0$. The expectation is that these divergences are canceled by counterterms. We will briefly expose this idea in the next subsection, without explaining it in full.

Let us write down explicitly the contributions $Y_{i}(G, \varphi, x)$ from loop graphs $G$ as in eq. (4.64). In $D$ dimensions, the formula is

$$
\begin{aligned}
Y_{i}(G, \varphi, x) & =\sum_{\substack{\text { leaf } \\
\text { orientations }}} \sum_{l_{e}, m_{e}, e \text { leaf }} \sum_{l_{e}, e \in G \backslash T}\left(\prod_{\text {vertices } v} \frac{1}{2 \pi \mathrm{i}} \int_{C_{v}} \frac{\mathrm{d} \delta_{v}}{\delta_{v}} \int_{S^{D-1}} \mathrm{~d} \Omega\left(\hat{y}_{v}\right)\right) \\
& \times \prod_{e \in T} \frac{\pi}{\sin \pi\left(l_{e}+\delta_{e}\right)} \mathrm{P}\left(-\hat{y}_{v} \cdot \hat{y}_{w}, l_{e}+\delta_{e}, D\right) \prod_{e \in G \backslash T} \mathrm{e}^{-\theta_{e} l_{e}} \mathrm{P}\left(\hat{y}_{v} \cdot \hat{y}_{w}, l_{e}, D\right) \\
& \times \exp \left(\ln r\left\{\sum_{\text {in leaves } e} l_{e}-\sum_{\text {out leaves } e}\left(l_{e}+D-2\right)+\sum_{\text {vertices } v}\left(2+\delta_{v}\right)\right\}\right) \\
& \times \prod_{\text {in leaves } e}\left(\prod_{v \succ e}\left(1+\epsilon_{v}\right)\right)^{-l_{e}-D+2} \\
& \times \prod_{\text {out leaves } e}\left(\prod_{v \succ e}\left(1+\epsilon_{v}\right)\right)^{D} \sqrt{\frac{D-2}{2 l_{e}+D-2}} h_{l_{e}, m_{e}}\left(\hat{y}_{e}\right) \mathbf{b}_{l_{e}, m_{e}}^{ \pm}:
\end{aligned}
$$


In the last line, we have set $\hat{y}_{e}=\hat{y}_{v}$ for an uncontracted leaf $e$ associated to some vertex $v$. The $\theta_{e}$ are defined as follows: If $v$ is a vertex, let

$$
\theta_{v}=\ln \prod_{w: w \succeq v}\left(1+\epsilon_{w}\right)
$$

and if $e=(v w) \in G \backslash T$, let $\theta_{e}=\theta_{v}-\theta_{w}$. The regulators are always chosen so that $\theta_{e}>0$, but evidently, $\theta_{e} \rightarrow 0$ as the regulators are removed.

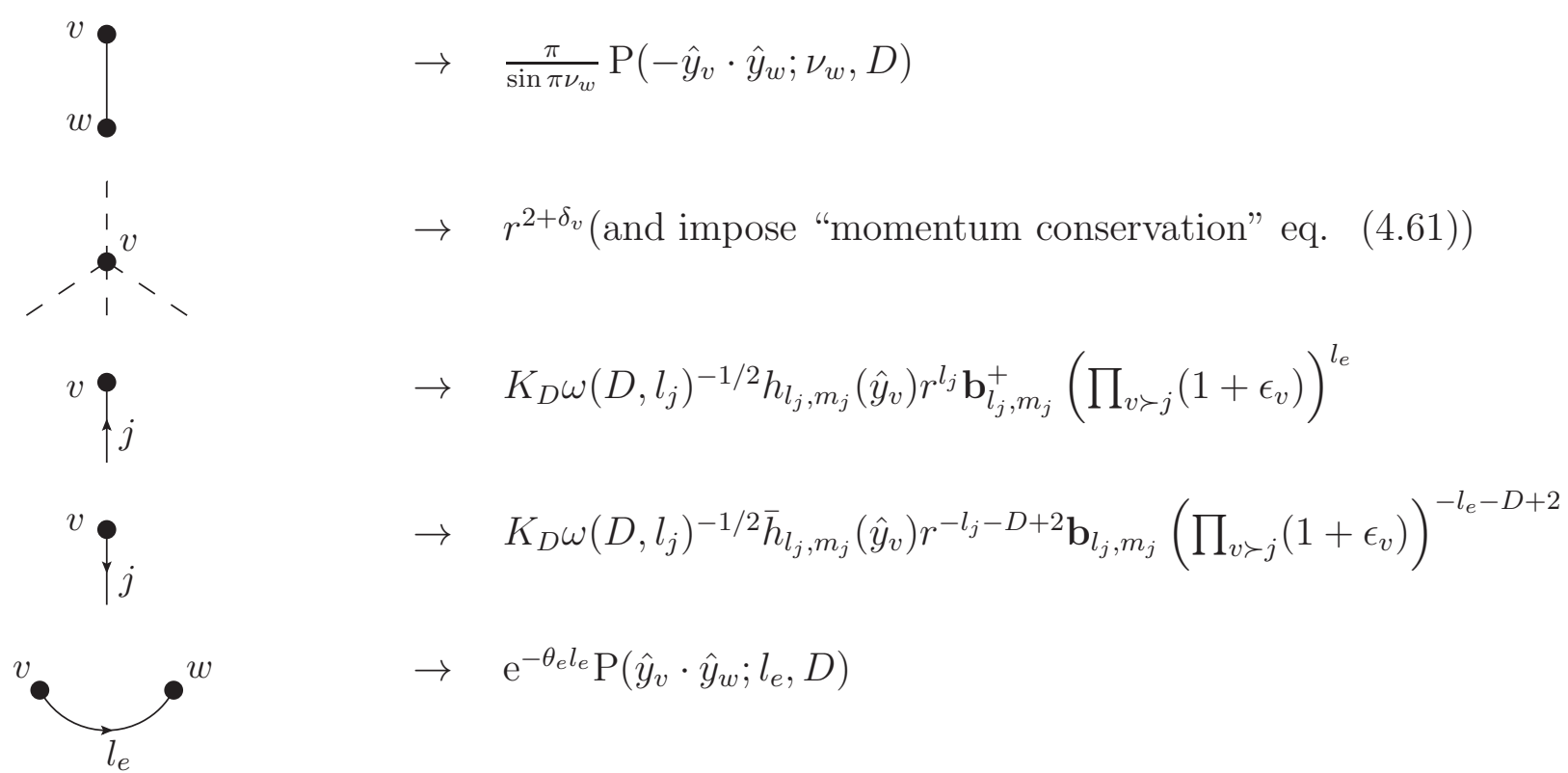

Figure 5: The rules for the "amplitude" of a graph in dimension $D \geq 3$. The appropriate summations and integrals have to be understood. As in fig. 4, we associate the powers of $r$ with the leaves and vertices instead of with the root.

It is possible to make the source of divergences more transparent by introducing the same trick as in $D=2$ to replace the summations over the angular momentum numbers $l_{e}$ by integrals. At each vertex, we have the momentum conservation rule eq. (4.66). As in $D=2$, we can realize this rule by introducing an integral similar to (4.67) at each such vertex. We can then perform the summations over the $l_{e}$ as we have done in eq. (4.72). The overall result of these manipulations is the following. Instead of the sum/integral in eq. (4.90), we now have

$$
\sum_{\substack{\text { leaf } \\ \text { orientations }}} \sum_{l_{e}, m_{e}, e \text { leaf }}\left(\prod_{\text {vertices } v} \int_{C_{v}} \frac{\mathrm{d} \delta_{v}}{\delta_{v}} \int_{S^{1} \times S^{D-1}} \mathrm{e}^{2 \mathrm{i} t_{v}} \mathrm{~d} t_{v} \wedge \mathrm{d} \Omega\left(\hat{y}_{v}\right)\right),
$$


i.e., we have gotten rid of the summation over the loop momenta, and replaced these by additional integrations over the parameters $t_{v}$. The term in the first product $(e \in T)$ in the second line of eq. (4.90) becomes, with $\beta_{e}:=\arccos \left(\hat{y}_{v} \cdot \hat{y}_{w}\right)$ for the line $e=(v w)$ connecting the vertices $v$ and $w$ :

$$
\begin{array}{r}
g_{D}\left(\delta_{e}, \cos \beta_{e}, t_{e}\right):=\frac{\pi}{\sin \pi \delta_{e}}\left(\sum_{l=0}^{\infty}(-1)^{l} \mathrm{e}^{\mathrm{i}\left(t_{e}+10\right) l} \mathrm{P}\left(\cos \beta_{e}, l+\delta_{e}, D\right)\right. \\
\left.+\sum_{l=-\infty}^{-1}(-1)^{l} \mathrm{e}^{\mathrm{i}\left(t_{e}-10\right) l} \mathrm{P}\left(\cos \beta_{e}, l+\delta_{e}, D\right)\right) .
\end{array}
$$

Putting together eq. (4.72) and eq. (B.146), we get for even $D$

$$
\begin{gathered}
g_{D}\left(\delta_{e}, \cos \beta_{e}, t_{e}\right)=\frac{\mathrm{e}^{-\mathrm{i} t_{e}(D / 2-1)}}{\Gamma(D / 2) \sin \pi \delta_{e}}\left(\frac{\partial}{2 \sin \beta_{e} \partial \beta_{e}}\right)^{\frac{D-2}{2}} \\
\times \quad\left(\mathrm{e}^{+\mathrm{i} \delta_{e} \beta_{e}} f\left(\delta_{e}, 1+\mathrm{e}^{\mathrm{i}\left(+\beta_{e}+t_{e}+\mathrm{i} 0\right)}\right)+\mathrm{e}^{\mathrm{i} \delta_{e} \beta_{e}} f\left(-\delta_{e}, 1+\mathrm{e}^{\mathrm{i}\left(-\beta_{e}-t_{e}+\mathrm{i} 0\right)}\right)\right. \\
\left.+\quad \mathrm{e}^{-\mathrm{i} \delta_{e} \beta_{e}} f\left(\delta_{e}, 1+\mathrm{e}^{\mathrm{i}\left(-\beta_{e}+t_{e}+\mathrm{i} 0\right)}\right)+\mathrm{e}^{-\mathrm{i} \delta_{e} \beta_{e}} f\left(-\delta_{e}, 1+\mathrm{e}^{\mathrm{i}\left(+\beta_{e}-t_{e}+\mathrm{i} 0\right)}\right)-\delta_{e}^{-1} \cos \left(\delta_{e} \beta_{e}\right)\right) .
\end{gathered}
$$

Here, $f$ is the transcendental function given by

$$
f(\delta, x)=\sum_{n=0}^{\infty} \frac{(\delta)_{n}}{n !}[\psi(n+1)-\psi(\delta+n)-\ln x] x^{n}
$$

also see eq. (B.144). For odd D, there is a similar formula obtained with the aid of theorem 2 of appendix B.

The term in the second product $(e \in G \backslash T)$ in eq. (4.90) is affected by the regulators. For these edges, we must replace the term in the second product over $(e \in G \backslash T)$ by the expression

$$
\sum_{l_{e} \in \mathbb{N}} \mathrm{e}^{\mathrm{i} l_{e}\left(t_{e}+\mathrm{i} \theta_{e}\right)} \mathrm{P}\left(\cos \beta_{e}, l_{e}, D\right)=\left[\left(\mathrm{e}^{\mathrm{i} \beta_{e}}-\mathrm{e}^{\mathrm{i}\left(t_{e}+\mathrm{i} \theta_{e}\right)}\right)\left(\mathrm{e}^{-\mathrm{i} \beta_{e}}-\mathrm{e}^{\mathrm{i}\left(t_{e}+\mathrm{i} \theta_{e}\right)}\right)\right]^{-(D-2) / 2} .
$$

There is also an additional factor of $\prod_{v:(v w)=e \in G \backslash T} \mathrm{e}^{\mathrm{i} t_{v}(D-2)}$ arising from the fact that the momentum conservation rule in $D$ dimensions is slightly different. The remaining parts of the formula (4.90) are unchanged. The total effect of these manipulations to eq. (4.90) is reflected in eq. (5.114) below.

The divergence that appears when we set $\epsilon_{v}=0$ in our graphical rules for the tree-like terms $Y_{i}(T, \varphi, x)$ now manifests itself as a pole of the transcendental function $f(\delta, x)$ at $x=0$, 
which corresponds to $\beta_{e}= \pm t_{e}-\pi$ in the above formula (4.94). More precisely, using the expansion of the function $f$ above, we can infer that the divergent behavior of $g_{D}$ are sums of terms of the form $\sim\left(t_{e} \pm \beta_{e}-\pi \pm \mathrm{i} 0\right)^{2-D}$. It can be seen from this that the most divergent part of the integrals that we have to consider is now given by an expression of the form

$$
I_{G}=\prod_{\text {vertices } v \text { in } G}\left(\int_{S^{D-1} \times S^{1}} \mathrm{~d} \Omega_{v} \mathrm{~d} t_{v}\right) \prod_{e \in T}\left|t_{e} \pm \beta_{e}-\pi\right|^{2-D} \prod_{e \in G \backslash T}\left|t_{e}+\mathrm{i} \theta_{e} \pm \beta_{e}\right|^{2-D} .
$$

In $D>3$, integrals of this type are no longer convergent for $\epsilon_{v}=0$ (i.e. $\theta_{e}=0$ ), but the divergences are very similar in nature to the divergences found in ordinary Feynman integrals in $x$-space. (But note that the " $x$ " space has become $S^{D-1} \times S^{1}$, and that the i0 prescriptions are different). In the usual approach to perturbation theory via Feynman integrals, these divergences have to be "renormalized" by hand. But in our approach the divergences are expected to cancel automatically when we include all contributions to $Y_{i}(\varphi, x)$ including the tree like terms and the counter terms before taking the regulators $\epsilon_{v}$ to zero. We now explain this in a little more detail.

\subsection{Renormalization}

In conventional perturbative quantum field theory, renormalization is necessary to make the terms in the perturbation series well-defined. In our approach the situation is somewhat different, because the consistency condition tells us in principle right from the start how to obtain well-defined perturbations of arbitrary order. We think this is a remarkable feature of the present framework. Nevertheless, we have already borrowed some vocabulary from renormalization such as "counterterms", and the reason for this is that we also need to perform various limits in our approach which are quite reminiscent of certain operations in conventional renormalization theory. In fact, the inclusion of the counterterms into the rules may be thought of as the "renormalization" of the tree-like contributions that we have treated in the last subsection and that diverge when the regulators are sent to 0 . The counterterms cure these divergences.

To state the "complete" set of rules is in principle straightforward, but generates a rather heavy notation. To keep the discussion reasonably transparent, we therefore only sketch the basic procedure, and leave a more detailed discussion to a future paper, where we will also outline the relation to Hopf-algebras similar to those in [7].

Let us go back to the start of the recursion procedure for evaluating $Y_{i}(\varphi, x)$. By eq. (4.79),

$$
Y_{i}(\varphi, x)=\frac{1}{3 !} G Y_{i-1}\left(\varphi^{3}, x\right)
$$


By eq. (4.80),

$$
\begin{aligned}
Y_{i-1}\left(\varphi^{3}, x\right) & =\sum_{j=0}^{i-1} Y_{j}(\varphi,(1+\epsilon) x) Y_{i-1-j}\left(\varphi^{2}, x\right) \\
& -\sum_{j=1}^{i-1} Y_{i-1-j}\left(Y_{j}(\varphi, \epsilon x) \varphi^{2}, x\right)-\frac{1}{|\epsilon x|^{D-2}} Y_{i-1}(\varphi, x)+\ldots
\end{aligned}
$$

where dots stand for terms vanishing for $\epsilon \rightarrow 0$. The counterterms (the terms with the --sign in front) can be rewritten as

$$
\begin{aligned}
& \sum_{j=1}^{i-1} Y_{i-1-j}\left(Y_{j}(\varphi, \epsilon x) \varphi^{2}, x\right)+\frac{1}{|\epsilon x|^{D-2}} Y_{i-1}(\varphi, x) \\
= & \sum_{j=1}^{i-1} \sum_{\operatorname{dim}(c) \leq 3(D-2) / 2}\left\langle c, Y_{j}(\varphi, \epsilon x) \varphi^{2}\right\rangle Y_{i-1-j}(c, x)+\frac{1}{|\epsilon x|^{D-2}} Y_{i-1}(\varphi, x)+\ldots
\end{aligned}
$$

where again, dots stand for terms vanishing for $\epsilon \rightarrow 0$. None of the terms in eq. (4.100) will give rise to tree-like summands in the final formula for $Y_{i}(\varphi, x)$. However, we can now apply the "incomplete" rules from the last subsection to the operators $Y_{j}(\varphi, \epsilon x), Y_{i-1-j}(c, x)$ and $Y_{i-1}(\varphi, x)$ appearing in eq. (4.100), and thus obtain more terms that contribute to $Y_{i}(\varphi, x)$. Graphically, we represent this by a "decorated tree" where the trees contributing to the matrix element $\left\langle c\left|Y_{j}(\varphi, \epsilon x)\right| \varphi^{2}\right\rangle$ are the decorations of a special vertex or blob, to which in turn the roots of the trees contributing to $Y_{i-1-j}(c, x)$ are attached ${ }^{6}$, see fig. 6 .

Of course we still do not have identified all the terms that make a contribution to $Y_{i}(\varphi, x)$. We would have to take into account the counterterms at each recursion step in the same manner as above. But we see that if we do so, we get all summands in the final regularized formula for $Y_{i}(\varphi, x)$ represented by (multiply) decorated trees. To summarize, we have laid out the following idea for calculating a vertex operator $Y_{i}(\varphi, x)$ :

- Draw a certain set of decorated trees with $i$ vertices.

- Apply a set of rules similar to those from subsec. 4.2.2 to each of these trees, translating them into an "amplitude" depending on a number of regulators $\epsilon_{v}$.

- Take the sum of all those amplitudes and take the limits $\epsilon_{v} \rightarrow 0$ in the appropriate order.

The development of rules for drawing decorated forests mentioned in the first bullet point above and the rules for amplitudes of decorated trees from the second bullet point is

\footnotetext{
${ }^{6}$ The term $|\epsilon x|^{2-D} Y_{i-1}(\varphi, x)$ would be represented in the same way by a blob decorated with the matrix element $|\epsilon x|^{2-D}=\left\langle\varphi\left|Y_{0}(\epsilon x)\right| \varphi^{2}\right\rangle$, to which a tree contributing to $Y_{i-1}(\varphi, x)$ is attached.
} 


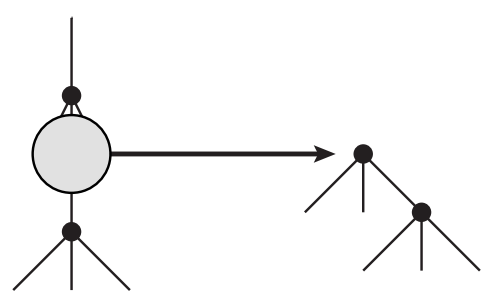

Figure 6: A decorated tree making a contribution to the vertex operator $Y_{4}(\varphi, x)$. More precisely, this tree makes a contribution to $G\left(\left\langle\varphi\left|Y_{2}(\varphi, \epsilon x)\right| \varphi^{2}\right\rangle Y_{1}(\varphi, x)\right)$; the tree pointed at by the arrow is a summand to $Y_{2}(\varphi, \epsilon x)$ and the tree below the "blob" is a summand to $Y_{1}(\varphi, x)$.

straightforward but cumbersome. They naturally lead to the appearance of a Hopf algebra structure similar to that found in [7] for ordinary Feynman diagrams. We will not state these rules here, leaving this to a future paper.

As an example, we consider the operator $Y_{2}(\varphi, x)$ for the theory with interaction $\lambda \varphi^{3} / 3$ ! in $D=4$. There are two trees with two 3 -valent vertices. We take the sum over all graphs spanned by these trees and include the one decorated tree that makes a contribution to the sum, see fig. 7 .
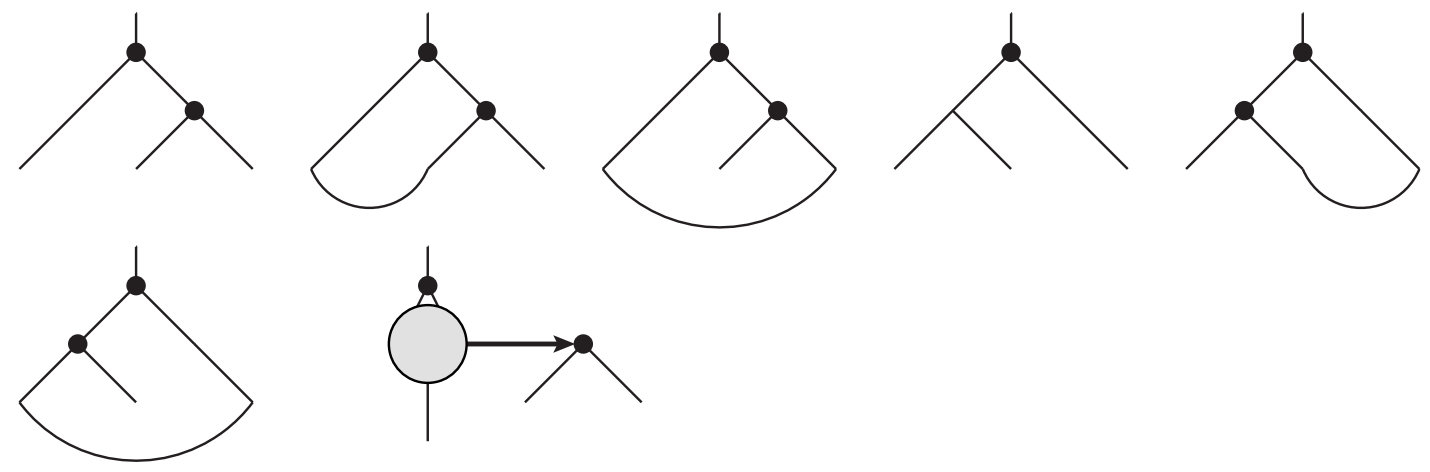

Figure 7: All trees contributing to the operator $Y_{2}(\varphi, x)$ for $D=4, P(\varphi)=\varphi^{3} / 3$ !. In the decorated tree, the tree pointed at by the arrow is contracted with the vectors $\langle\varphi|,| \varphi\rangle$. 
The contribution of the decorated tree is

$$
\begin{aligned}
& \sum_{l, m}\left(\frac{1}{2 \pi \mathrm{i}}\right)^{2} \oint_{C_{1}} \frac{\mathrm{d} \delta_{1}}{\delta_{1}} \oint_{C_{2}} \frac{\mathrm{d} \delta_{2}}{\delta_{2}} h_{l, m}(\hat{x}) \omega(4, l)^{-1 / 2} \mathbf{b}_{l, m} \\
\times & \frac{\epsilon^{\delta_{2}}}{\delta_{2}\left(2+\delta_{2}\right)} \frac{r^{l+\delta_{1}+\delta_{2}+2}}{4 l+2 l\left(\delta_{1}+\delta_{2}\right)+8+6\left(\delta_{1}+\delta_{2}\right)+\left(\delta_{1}+\delta_{2}\right)^{2}}
\end{aligned}
$$

Here, we have already performed the (trivial) integrals over the angular variables and we have not used the trick (4.85) because the sums over the "momenta" of internal lines are trivial as well. In the limit $\epsilon \rightarrow 0$, the expression (4.101) is logarithmically divergent, as can be seen from evaluating the contour integral in $\delta_{2}$. This has to be compared with the divergences in the loop graphs. Consider the contribution from the second tree in the first line of fig. 7 :

$$
\begin{aligned}
& \sum_{l, m, l_{1}}\left(\frac{1}{2 \pi \mathrm{i}}\right)^{2} \oint_{C_{1}} \frac{\mathrm{d} \delta_{1}}{\delta_{1}} \oint_{C_{2}} \frac{\mathrm{d} \delta_{2}}{\delta_{2}} \int_{S^{3}} \mathrm{~d} \Omega\left(\hat{y}_{1}\right) \int_{S^{3}} \mathrm{~d} \Omega\left(\hat{y}_{2}\right) \\
\times & \mathrm{P}\left(\hat{y}_{1} \cdot \hat{y}_{2}, l_{1}, 4\right) \frac{\pi}{\sin \pi \delta_{2}} \mathrm{P}\left(\hat{y}_{1} \cdot \hat{y}_{2}, l-l_{1}+\delta_{2}, 4\right)(1+\epsilon)^{l-l_{1}+\delta_{2}} \\
\times & \frac{\pi}{\sin \pi\left(\delta_{1}+\delta_{2}\right)} \mathrm{P}\left(\hat{y}_{1} \cdot \hat{x}, l+2+\delta_{1}+\delta_{2}, 4\right) \\
\times \quad & r^{l+2+\delta_{1}+\delta_{2}} h_{l, m}\left(\hat{y}_{2}\right) \omega(4, l)^{-1 / 2} \mathbf{b}_{l, m}^{+}
\end{aligned}
$$

We have only kept track of one regulator $\epsilon$; the other regulators $\epsilon_{v}$ that one would obtain by following the graphical rules by the letter give rise to trivial limits $\epsilon_{v} \rightarrow 0$.

The contributions from the other loop graphs are similar. In the loop graphs, the divergences arise from the loop summations which is the sum over $l_{1}$ in eq. (4.102). These divergences should be canceled by the counterterm (4.101). In the previous section, we have applied manipulations which turned the sums into integrals, and the divergences then manifest themselves at the level of the integrals $I_{G}$, as we discussed. It is still not an easy matter to see that the counterterms precisely cancel the divergence, already in this example. See [13] for a detailed discussion of examples of that nature. It seems that a different method is required to make such cancellations manifest in a systematic way.

Counterterms also occur in the construction of composite operators. Here, what have been "tree-like summands" before become "forest-like summands". For an operator $Y_{i}(a, x)$ these would have a graphical representation by forests consisting of $|a|$ trees with $i$ vertices overall. The rules for the contribution to $Y_{i}(a, x)$ of such a forest are more or less the same as the rules from section 4.2.2 applied to every tree in the forest. Again, the notation necessary to state the rules in full, including decorated forests for the counterterms, is quite heavy, and we will not spell this out here. 


\section{$5 \quad$ Vertex algebra and special functions}

In this section, we will give yet another representation of the vertex operator $Y(\varphi, x)$ in the interacting field theory characterized by the interaction $\lambda P(\varphi)=\lambda \sum \frac{c_{p}}{p !} \varphi^{p}$. We believe that this representation is interesting not only because it might be convenient for calculations, but also because it hints at a deep relation between our vertex algebras and certain special functions of hypergeometric type.

Our starting point is the representation of $Y(\varphi, x)=\sum_{i} \lambda^{i} Y_{i}(\varphi, x)$ in the form of eqs. (4.65) and (4.90). We further decompose the $i$-th order contribution $Y_{i}(\varphi, x)$ to the vertex operator into contributions from counterterms (when $D>2$ ), and from graphs, $G$, as outlined above, and these are called again $Y_{i}(G, \varphi, x)$. The main point of this section is to provide an alternative representation for this quantity. Since each $Y_{i}(G, \varphi, x)$ is an endomorphism in $\operatorname{End}(V)$, it is sufficient to present a formula for the matrix elements in a basis of $V$.

To present our result in the most economical form, it is convenient to choose a particular (non-orthogonal) basis of $V$ which is defined as follows. First, for any $p \in \mathbb{R}^{D}, l \in \mathbb{N}$, we define $\mathbf{b}_{l}(p)^{+}=\omega(l, D)^{-1 / 2} \sum h_{l, m}(p) \mathbf{b}_{l, m}^{+}$. For $\vec{p}=\left(p_{1}, \ldots, p_{n}\right) \in \mathbb{R}^{n D}$ and $\vec{l}=\left(l_{1}, \ldots, l_{n}\right) \in \mathbb{N}^{n}$ we then define

$$
|\vec{p}, \vec{l}\rangle:=\prod_{i=1}^{n} \mathbf{b}_{l_{i}}\left(p_{i}\right)|0\rangle .
$$

We remember that any element in $V$ corresponds to a composite field, i.e., a formal product of $\varphi$ and its derivatives. For the vectors just defined, this is

$$
|\vec{p}, \vec{l}\rangle=\prod_{i=1}^{n} \mathrm{P}\left(p_{i} \cdot \partial, l_{i}, D\right) \varphi
$$

It is evident from this expression that the vectors $|\vec{p}, \vec{l}\rangle$ form an (overcomplete) basis of $V$. Our aim in this section is to provide an alternative expression for the matrix elements $\left\langle\vec{p}_{-}, \vec{l}_{-}\left|Y_{i}(G, \varphi, x)\right| \vec{p}_{+}, \vec{l}_{+}\right\rangle$.

Our starting point is the formula (4.90) for the $Y_{i}(G, \varphi, x)$. The basic idea is to carry out the angular integrals $\int \mathrm{d} \Omega\left(\hat{y}_{v}\right)$ first, or rather, to turn these integrals into summations. This is done by first expanding the Legendre functions using the formula (valid for $\nu \notin \mathbb{Z}$ )

$$
\mathrm{P}(z, \nu, D)=\frac{\sin \pi \nu}{\pi} \frac{2^{-(D+1) / 2}}{\Gamma(D / 2)} \sum_{n=0}^{\infty}(-2 z)^{n} \frac{\Gamma(-\nu / 2+n / 2) \Gamma(\nu / 2+n / 2+D / 2-1)}{n !} .
$$

which we prove in the appendix B. For $\nu=l \in \mathbb{Z}$, we have instead the well-known formula

$$
\mathrm{P}(z, l, D)=\frac{2^{-(D-3) / 2}}{\Gamma(D / 2)} \sum_{j=0}^{[l / 2]}(-2 z)^{l-2 j} \frac{\Gamma(l-j+D / 2-1)}{j !(l-2 j) !} .
$$


If we perform these expansions for all the Legendre functions appearing in eq. (4.90), then we end up with a multiple sum, whose terms contain powers $\left(\hat{y}_{v} \cdot \hat{y}_{w}\right)^{n_{e}}$, where $e=(v w)$ is an edge between $v, w$, and where each $n_{e}$ is the summation counter from the power series expansion of the Legendre functions associated with the edge $e$. To perform the angular integrals, we now further expand each such power using the multinomial formula,

$$
\left(\hat{y}_{v} \cdot \hat{y}_{w}\right)^{n_{e}}=\sum_{k_{e, 1}+\cdots+k_{e, D}=n_{e}} \frac{n_{e} !}{\prod_{\mu} k_{e, \mu} !} \prod_{\mu}\left(\hat{y}_{v, \mu} \hat{y}_{w, \mu}\right)^{k_{e, \mu}} .
$$

Here, and in the following, $\mu$ runs from 1 to $D$. After the combined expansions, each term in eq. (4.90) will now consist of a prefactor times $\hat{y}_{v, \mu}$, raised to some power $a_{v, \mu}$. The power is

$$
a_{v, \mu}=\sum_{e \text { adjacent } v} k_{e, \mu},
$$

where the sum is over all edges $e$ going from the vertex $v$. Thus, the integrals we have to consider are of the type $\left(a_{i} \in \mathbb{N}\right)$

$$
\int_{S^{D-1}} \mathrm{~d} \Omega(\hat{x}) \hat{x}_{1}^{a_{1}} \cdots \hat{x}_{D}^{a_{D}}= \begin{cases}2 \frac{\prod_{\mu} \Gamma\left(\frac{a_{\mu}+1}{2}\right)}{\Gamma\left(\frac{\sum_{\mu} a_{\mu}+D}{2}\right)} & \text { if all } a_{i} \text { even } \\ 0 & \text { otherwise }\end{cases}
$$

This formula can be viewed as a multi-dimensional generalization of the standard formula for the Euler Beta-function $(D=2)$ and can be proved e.g. by induction in $D$, expressing $\mathrm{d} \Omega$ in $D$-dimensional polar coordinates. If we combine all the steps we have described so far, then we end up with the following expression (up to a numerical prefactor) for the vertex operator:

$$
\begin{aligned}
& \left\langle\vec{p}_{-}, \vec{l}_{-}\left|Y_{i}(G, \varphi, x)\right| \vec{p}_{+}, \vec{l}_{+}\right\rangle= \\
& r^{2 i} \sum_{\substack{\text { assignments } \\
\vec{l}_{+}, \vec{l}_{-} \rightarrow \text { leaves }}} \sum_{\substack{e \in \mathbb{N}: e \in G \backslash T \\
k_{e} \in \mathbb{N}^{D}: e \in T}}\left(\prod_{v \in T} \frac{1}{2 \pi \mathrm{i}} \int_{C_{v}} \frac{\mathrm{d} \delta_{v}}{\delta_{v}}\right) \\
\times & \prod_{e \in T} \frac{\Gamma\left(-l_{e} / 2-\delta_{e} / 2+\left|k_{e}\right| / 2\right) \Gamma\left(l_{e} / 2+\delta_{e}+D / 2-1+\left|k_{e}\right| / 2\right)}{k_{e} !} \\
\times & \prod_{v \in T} \frac{\prod_{\mu} \Gamma\left(\left(\sum_{e \text { on } v} k_{e, \mu}+1\right) / 2\right)}{\Gamma\left(\left(\sum_{e \text { on } v}\left|k_{e}\right|+D\right) / 2\right)} \prod_{e \in G \backslash T} \frac{\Gamma\left(l_{e}-j_{e}+D / 2-1\right)}{j_{e} !\left(l_{e}-2 j_{e}\right) !} \\
\times & \prod_{e \text { in }} \frac{\Gamma\left(l_{+e}-j_{e}+D / 2-1\right)}{j_{e} !\left(l_{+e}-2 j_{e}\right) !} \prod_{e \text { out }} \frac{\Gamma\left(l_{-e}-j_{e}+D / 2-1\right)}{j_{e} !\left(l_{-e}-2 j_{e}\right) !} \\
\times & \hat{x}^{k_{0}} r^{\sum_{e \text { in }} l_{+e}-\sum_{e \text { out }}\left(l_{-e}-D+2\right)+\sum_{v \in T} \delta_{v}}(-2)^{\sum_{e}\left|k_{e}\right|} \prod_{e \text { in }} p_{+e}^{k_{e}} \prod_{e \text { out }} p_{-e}^{k_{e}} \prod_{e \in G \backslash T} \mathrm{e}^{-l_{e} \theta_{e}}
\end{aligned}
$$


Eq. (5.111) requires several comments. First, we have the regularizing factors from eq. (4.90). This means that eq. (5.111) rigourously makes sense in arbitrary $D$, but for $D \geq 3$ the expression is divergent if we let $\epsilon_{v} \rightarrow 0$. We have left out the regularizing factors associated with uncontracted leaves because they remain regular in the limit $\epsilon_{v} \rightarrow 0$. As above, $T$ is a tree on $1, \ldots, i$, and $G$ is a graph from $\mathcal{G}(T)$, the set of all loop graphs for which $T$ is a spanning tree, and which result from $T$ by joining leaves together and hence forming loops. The edges are $e$, and the vertices $v$. The leaves on $T$ that were joined to form loops are hence $e \in G \backslash T$, and with each of them, we have a summation counter $l_{e} \in \mathbb{N}$. The $l_{e}$ 's associated with the uncontracted leaves are assigned to the entries in $\vec{l}_{ \pm}$. Here, the notation " $e$ in" means that $e$ runs through the incoming leaves, while " $e$ out" that it runs through the outgoing leaves. The $l_{e}$ associated with the incoming leaves is set equal to an $l_{+e}$ in the vector $\vec{l}_{+}$, while the $l_{e}$ associated with the outgoing leaves is set equal to an $l_{-e}$ in the vector $\vec{l}_{-}$. By the first summation sign in the first line of eq. (5.111), we mean the sum over all possible assignments of the entries of $\vec{l}_{+}, \vec{l}_{-}$to the uncontracted leaves of $G$. A similar notation is used above for the vectors $\vec{p}_{+}, \vec{p}_{-}$.

The $l_{e}$ associated with edges that are neither leaves, nor contracted leaves are referred to as " $e \in T$ ", and if $e=(v w)$ they are also called $l_{v}$ in the last line. The value of $l_{e}$ on such an edge is determined by the momentum conservation rule (4.66). Furthermore, with each edge, we have additional summation counters $k_{e} \in \mathbb{N}^{D}$. The sum over $k_{e}$ when $e \in G \backslash T$ is over those integer vectors for which $2 j_{e}:=l_{e}-\left|k_{e}\right|$ is even. For such integer vectors $k=\left(k_{\mu}\right) \in \mathbb{N}^{D}$, and vectors $p=\left(p_{\mu}\right) \in \mathbb{R}^{D}$ we use the following multi-index notation:

$$
|k|=\sum_{\mu} k_{\mu}, \quad p^{k}=\prod_{\mu} p_{\mu}^{k_{\mu}}, \quad k !=\prod_{\mu} k_{\mu} ! .
$$

We write " $e$ on $v$ " to mean that the sum/product is running over those edges $e$ going out from the vertex $v$. The integer vector $k_{0} \in \mathbb{N}^{D}$ is the counter associated with the root $e=0$.

Formula (5.111) is the desired alternative representation of the contribution to the vertex operator from an individual graph $G$. In $D=2$, the full vertex operator is given by the sum of the graph contributions (5.111) with $\epsilon_{v}=0$, as in eq. (4.64). For $D \geq 3$, we have to keep the regulators $\epsilon_{v}$ finite. The full vertex operator is the sum of the contributions from the graphs (5.111) and the counterterms whose construction was outlined in sec. 4.3. The limit as $\epsilon_{v} \rightarrow 0$ has to be performed in the end.

The residue integrals $\int \mathrm{d} \delta_{v} / \delta_{v}$ can be performed straightforwardly using the well-known Laurent expansion of the Gamma-function around integer values, which can be inferred from the standard formula

$$
\Gamma(1+\delta)=\frac{1}{1+\delta} \mathrm{e}^{\delta\left(1-\gamma_{\mathrm{E}}\right)} \exp \left\{\sum_{n=2}^{\infty}(-\delta)^{n}\left(\zeta_{n}-1\right) / n\right\},
$$

where $\zeta_{n}$ are the values of the Riemann Zeta-function ${ }^{7}$. Thus, we see that we get a repre-

\footnotetext{
${ }^{7}$ We expect to see a connection to the " $Z$-sums" described e.g. in [27] and their algebra when performing
} 
sentation involving only (multiple) sums.

It is worth contrasting the above formula (5.111) with the integral representations for the contribution $Y_{i}(G, \varphi, x)$ that were derived earlier in sec. 4. For comparison, we here repeat these formula, or more precisely, the matrix elements of them. In doing so, we take the opportunity to replace the integrals $\prod_{v} \int_{S^{D-1}} \mathrm{~d} \Omega\left(\hat{y}_{v}\right)$ over the spheres by integrals over the corresponding $S O(D)$-invariants $\hat{y}_{v} \cdot \hat{y}_{w}$, using a general formula due to [10]. More precisely, let us write $\left\{\vec{p}_{+}, \vec{p}_{-}\right\}=\left\{p_{i} \mid i=1, \ldots, L\right\}$ for the "momenta" associated with the states $\left|\vec{p}_{ \pm}, \vec{l}_{ \pm}\right\rangle$. We order the vertices so that the vertex $v=0$ is the root, so that the vertices $v=1, \ldots, L$ correspond to the leaves, and so that the internal vertices are $v=L+1, \ldots, L+i+1$. Then, for each pair $(v w)$ of vertices we introduce a real integration variable $z_{v, w}$, and if $(v w)=e$ represents an edge $e$ of the graph $G$, we also write $z_{v, w}=z_{e}$. From these quantities, we also define the following symmetric $D \times D$ matrices:

$$
Z_{D, k}=\left(\begin{array}{cccc}
z_{0,0} & \cdots & z_{D-2,0} & z_{k, 0} \\
\vdots & & \vdots & \vdots \\
z_{0, D-2} & \cdots & z_{D-2, D-2} & z_{k, D-2} \\
z_{0, k} & \cdots & z_{D-2, k} & z_{k, k}
\end{array}\right)
$$

The formulae derived in sec. 4 can then be seen to take the form in $D>2$ (up to numerical factors):

$$
\begin{aligned}
& \left\langle\vec{p}_{-}, \vec{l}_{-}\left|Y_{i}(G, \varphi, x)\right| \vec{p}_{+}, \vec{l}_{+}\right\rangle=r^{2 i+\sum_{e \text { in }} l_{+e}-\sum_{e \text { out }}\left(l_{-e}+D-2\right)} \\
& \times \sum_{\substack{\text { assignments } \\
\vec{l}_{+}, \vec{l}_{-} \rightarrow \text { leaves }}}\left(\prod_{L+1 \leq v \leq L+i+1} \frac{1}{2 \pi \mathrm{i}} \int_{C_{v}} \frac{\mathrm{d} \delta_{v}}{\delta_{v}} r^{\delta_{v}}\right) \\
& \times \prod_{1 \leq v \leq L+i+1}\left(\int_{0}^{2 \pi} \mathrm{e}^{2 \mathrm{i} t_{v}} \mathrm{~d} t_{v}\right) \int_{\mathscr{M}_{D, L+i+1}} \prod_{L+1 \leq v \leq L+i+1} \frac{1}{\sqrt{\operatorname{det}\left(Z_{D, v}\right)}} \prod_{\substack{0 \leq v \leq D-2 \\
v<w \leq L+i+1}} \mathrm{~d} z_{w, v} \\
& \times \quad \prod_{1 \leq v \leq L} \delta\left(\hat{x} \cdot p_{v}-z_{0, v}\right) \prod_{\substack{1 \leq v \leq D-2 \\
v<w \leq L}} \delta\left(p_{v} \cdot p_{w}-z_{v, w}\right) \\
& \times \quad \prod_{e \in T} g_{D}\left(\delta_{e}, z_{e}, t_{e}\right) \prod_{e \in G \backslash T}\left[\left(\mathrm{e}^{\mathrm{i} \arccos z_{e}}-\mathrm{e}^{\mathrm{i}\left(t_{e}+\mathrm{i} \theta_{e}\right)}\right)\left(\mathrm{e}^{-\mathrm{i} \arccos z_{e}}-\mathrm{e}^{\mathrm{i}\left(t_{e}+\mathrm{i} \theta_{e}\right)}\right)\right]^{-(D-2) / 2} \\
& \times \quad \prod_{e \text { in }} \mathrm{P}\left(z_{e}, l_{+e}, D\right) \prod_{e \text { out }} \mathrm{P}\left(z_{e}, l_{-e}, D\right) \prod_{1 \leq v \leq L_{+}} \mathrm{e}^{\mathrm{i} t_{v}(D-2)} .
\end{aligned}
$$

Here, $g_{D}$ are the distributions given explicitly in eq. (4.94), and the $\delta$ 's in the fourth line are Dirac $\delta$-distributions. In $D=2$, the terms in the last product are replaced by $\log$ 's.

the residue in eq. (5.111). 
Furthermore, $\mathscr{M}_{D, n}$ is the manifold of dimension $(r-1)(n-r / 2)$ of all real, positive $n \times n$ matrices $M=\left(M_{i j}\right)$ of rank $r=\min (D, n)$ such that $M_{i i}=1$. Thus, we see that while formula (5.111) is given entirely in terms of infinite sums, the above representation (5.114) is entirely in terms of convergent integrals. The integral over $\mathscr{M}_{D, L+i+1}$ will become divergent for $D \geq 3$ in the limit when the regulators are removed, the divergences coming from the boundary $\partial \mathscr{M}_{D, L+i+1}$. As was the case for the previous formula, for $D \geq 3$, we would have to calculate the vertex operator $\left\langle\vec{p}_{-}, \vec{l}_{-}\left|Y_{i}(\varphi, x)\right| \vec{p}_{+}, \vec{l}_{+}\right\rangle$as the sum of all the above terms $\left\langle\vec{p}_{-}, \vec{l}_{-}\left|Y_{i}(G, \varphi, x)\right| \vec{p}_{+}, \vec{l}_{+}\right\rangle$plus the counterterms, before removing the regulators. This was outlined in sec. 4.3 .

It is fair to ask what is the value of having the alternative representations (5.111) and (5.114). It is not clear that either representation has much of an advantage computationally, as there is essentially an equivalent number of summations as there are integrations in both formulae (5.111) and (5.114). However, the alternative representation (5.111) brings out a striking feature that was far from obvious when we started the construction of the vertex operators, namely that it can be represented in terms of (multiple) infinite series of a very special form, with each term being a monomial times a ratio of Gamma-functions. Because of this feature, the above series can be viewed as a generalization of the Gauss hypergeometric series, and the particular form of the series is governed by the graph $G$ under consideration. The vertex operators $Y_{i}(a, x)$ may also be defined for general $a \in V$, and their matrix elements have a similar representation. Furthermore, the vertex operators satisfy the consistency relation (2.9). We expect that this relation will give highly non-trivial relations between the above functions of hypergeometric type. Those relations make this class of multi-variate functions special. By analyzing the relations that are obtained in more detail, we expect that one can uncover interesting relations between our vertex operator algebras and the theory of special functions. We think that this is a fruitful direction for further research.

It remains to be understood better how to incorporate the renormalization procedure into this approach. As we have emphasized before, the above formula only yields a convergent result for $D=2$, while it is only an incomplete representation of the vertex operator for $D>2$. It appears that a formula of the above nature including renormalization can also be given, but this involves the use of a Hopf-algebra structure similar to that of [7]. We will pursue this in another paper as well.

\section{Relation to vertex operator algebras in CFT's}

Vertex operator algebras have been discussed previously in the literature (see e.g. $[3,9,19]$ ) in the context of conformally invariant QFT's (CFT's), so we explain the difference between our framework/motivation and those approaches.

First of all, we stress again our framework is in essence a "repackaging" of the information contained in the Wilson operator product expansion (OPE), which is a standard tool in 
QFT. The relation is, as we explained above, $\langle c|Y(a, x)| b\rangle=C_{a b}^{c}(x)$. Our new realization is that, when repackaged in this way, the OPE satisfies properties that can be encoded in some kind of vertex algebra. As we have discussed, this repackaging allows both for a new conceptual viewpoint of perturbation theory via Hochschild cohomology and a new way to do calculations in perturbative QFT.

By contrast to the vertex operator algebras that have previously been considered in the context of conformally invariant theories in $D=2$ dimensions, our approach is intended to work not just for 2-dimensional CFT's, but for any QFT (in any dimension) whose Schwinger functions have an OPE. Despite this key difference, there are some evident parallels: As in our approach, one considers endomorphisms $Y(a, x) \in \operatorname{End}(V)$, where $a \in V$. Also, similar to our approach, the vectors $a$ are interpreted both as states and as fields ("state-field correspondence"), and the $Y(a, x)$ satisfy certain properties that are similar to ours. More precisely, in the usual approaches, $\mathbb{R}^{2}$ is identified with $\mathbb{C}$, and the vertex operators are formal distributions ${ }^{8}, Y(a,.) \in \operatorname{End}(V) \otimes \mathbb{C}\left[\left[x, x^{-1}\right]\right]$, where $\mathbb{C}\left[\left[x, x^{-1}\right]\right]$ is the ring of formal sums of the form $\sum_{k \in \mathbb{Z}} a_{k} x^{k}$ with complex coefficients. The analogue of the transformation formula under $S O(2)$ is played by a formula expressing the conformal invariance of the theory, i.e. either under $P S L_{2}(\mathbb{R})$ or even the Virasoro algebra. A notion of vertex algebra in $D$ dimensions that is in a similar spirit and is applicable to globally conformally invariant theories has also been introduced, see [24]. The appearance of the ring of formal Laurent series in the CFT context is connected in an essential way to the fact that, in conformally invariant theories, the OPE-coefficients only have singularities of a very special form due to conformal invariance.

Our consistency condition, as stated above in eq. (1.5), does not make sense as it stands in the standard CFT-vertex algebra context [9, 19], because the left side of the condition would be an element in the $\operatorname{ring} \operatorname{End}(V) \otimes \mathbb{C}\left[\left[x, x^{-1}, y, y^{-1}\right]\right]$, whereas the right side would be an element in the ring $\operatorname{End}(V) \otimes \mathbb{C}\left[\left[(x-y),(x-y)^{-1}\right]\right]\left[\left[y, y^{-1}\right]\right]$, and there is no natural way to identify these rings without some notion of convergence. Such a notion of convergence is available (and used) in our context, because we deal with the ring of holomorphic functions, but it is not usually considered in the CFT-context, where one prefers to work with the above rings of formal series. A related problem with our form of the consistency condition in the CFT-context is that the condition $0<|x-y|<|y|<|x|$, which is essential for our form of the consistency condition, does not even make sense in the ring of formal series considered in the CFT context.

However, in the CFT context, there is another way to formulate a consistency condition which bypasses any convergence considerations, and which leads to a relation with a superficially similar appearance. This condition can be stated in various equivalent ways, but a particularly transparent formulation is that for each $a, b \in V$ there is an $N \in \mathbb{N}$ such that

$$
(x-y)^{N}[Y(a, x) Y(b, y)-Y(b, y) Y(a, x)]=0 .
$$

\footnotetext{
${ }^{8}$ To be precise, our vertex operators should be written $Y(a, x, \bar{x})$, because they are not holomorphic in $x$ unlike in the CFT context.
} 
This equation now makes sense in the CFT context as an equation in the $\operatorname{ring} \operatorname{End}(V) \otimes$ $\mathbb{C}\left[\left[x, x^{-1}, y, y^{-1}\right]\right]$, but it no longer makes sense in our context: The first term would be defined in the domain $|x|>|y|$, whereas the second term would be defined in the disjoint domain $|y|>|x|$. Thus, the situation with regards to our consistency condition (1.5) and with (6.115) is exactly opposite: Our condition (1.5) cannot even be formulated in the standard vertex algebra approach in CFT's, while eq. (6.115) cannot even be formulated in our approach. While one can derive from eq. (6.115) an equation that does make sense in the CFT-context, and that is similar in appearance to our consistency condition (1.5),

$$
(x-y)^{N} Y(a, x-y) Y(b,-y) c=(x-y)^{N} Y(Y(a, x) b,-y) c,
$$

see [19, Lemma 4.6], one sees that there are notable differences between this and our consistency condition ${ }^{9}$.

The equation (6.115), which is often called "locality" in the CFT-vertex operator algebra context, may also be restated as saying that the commutator $[Y(a, x), Y(b, y)]$ is a finite sum of derivatives of "formal delta distributions" 10 in the sense that

$$
[Y(a, x), Y(b, y)]=\sum_{n=0}^{N-1}\left[\partial^{n} \delta\right](x-y) Y\left(c_{n}, y\right), \quad a, b, c_{n} \in V .
$$

Especially when written in the last form one can understand that the "locality condition" is intimately related to the fact that, in 2-dimensional CFT's, the commutator of local operators are supported on the lightcone (in a Minkowski formulation of the theory) - or equivalently - that the singularities of the OPE coefficients are of the form $(x-y)^{-n}$, where $n \in \mathbb{N}$ (in a Euclidean formulation of the theory). By contrast, the singularities in a generic QFT do not have to be of this form, and may e.g. contain log's, as exemplified by the perturbative constructions of this paper. Furthermore, in a generic QFT, it is no longer true that the terms in the OPE are smooth apart from a finite number of terms, as even terms in the OPE

$$
\mathcal{O}_{a}(x) \mathcal{O}_{b}(0)=\sum_{c} C_{a b}^{c}(x) \mathcal{O}_{c}(0)
$$

corresponding to fields $\mathcal{O}_{c}$ of arbitrarily large dimension still typically contain log's and are thus not strictly smooth.

For these reasons, we are somewhat pessimistic that a fruitful definition of vertex algebra of the same purely algebraic flavor as described e.g. in [19] can also be found for the generic

\footnotetext{
${ }^{9}$ In the approach [11, Sec. 3.4] to CFT's (see also references therein) the consistency condition is the same as ours. However, he does not impose the restriction $0<|x-y|<|y|<|x|$ for the validity of the equation. This feature is accidental in CFT and essentially due to the fact that three points $0, x, y \in \mathbb{C}$ can be brought into an arbitrary relative position by a conformal transformation, but not by a Euclidean transformation. Hence, also the underlying reasoning leading to the consistency condition as given in this reference is specific to CFT's and cannot be repeated in more generic situations.

${ }^{10}$ The formal delta distributions are defined as the formal series $\delta(x-y)=\sum_{n \in \mathbb{Z}} x^{n} y^{-n-1}$.
} 
(e.g., perturbative) QFT models. However, we think that this direction deserves further study, and some ideas in this direction have been put forward by [4].

Acknowledgements: We would like to thank R. Brunetti, J. Holland, N. Nikolov, and I. Runkel for discussions. We would also like to thank R. Brunetti for pointing out to us ref. [10]. The work of H. Olbermann was supported by an EPSRC Doctoral Training Grant.

\section{A Spherical harmonics and Legendre functions in $D$ dimensions}

Polynomials $h(x), x \in \mathbb{R}^{D}$ which are solutions to the Laplace equation $\Delta h(x)=0$ are called "harmonic polynomials". Since the Laplace operator $\Delta$ commutes with dilations $x \mapsto t x$, it follows that any harmonic polynomial can be decomposed into a sum of homogeneous harmonic polynomials. The harmonic polynomials satisfying $h(t x)=t^{l} h(x), l \in \mathbb{N}$ span a vector subspace of dimension $N(l, D)$ in $\mathbb{C}[x]$, where $N(0, D)=1$ and

$$
N(l, D)=\frac{(2 l+D-2)(l+D-3) !}{(D-2) ! l !} \text { for } l>0 .
$$

This can be seen for example by noting that the degree $l$ harmonic polynomials $h(x)$ are in one-to-one correspondence with totally symmetric, traceless tensors of rank $l$ on $\mathbb{R}^{D}$ : If $c_{\mu_{1} \ldots \mu_{l}}$ are the components of such a tensor, then $h(x)=\sum c_{\mu_{1} \ldots \mu_{l}} x_{\mu_{1}} \cdots x_{\mu_{l}}$ is a harmonic polynomial of degree $l$, and vice versa. The spherical harmonics in $D$ dimensions are by definition the restrictions of the harmonic polynomials to $S^{D-1}$.

In the main text, we consider the a basis $h_{l, m}(x), m \in\{1, \ldots, N(D, l)\}$ of degree $l$ harmonic polynomials for each $l \in \mathbb{N}$. The members of this basis are chosen to satisfy the orthogonality condition eq. (3.28). In fact, the $h_{l, m}$ form an orthonormal basis of $L^{2}\left(S^{D-1}, \mathrm{~d} \Omega\right.$ ) when restricted to the sphere. It follows immediately from the fact that the $h_{l, m}(x)$ are harmonic polynomials that their restrictions $h_{l, m}(\hat{x})$ to the sphere are eigenfunctions of the Laplacian $\hat{\Delta}$ on $S^{D-1}$ with eigenvalue $-l(l+D-2)$.

For our calculations in appendix $\mathrm{C}$, we need to know in more detail the relation of the harmonic polynomials $h_{l, m}$ to the traceless symmetric tensors of rank $l$ described above. To state the relevant facts, we use the familiar multi-index notation, $\alpha=\left(\alpha_{1}, \ldots, \alpha_{D}\right) \in \mathbb{N}^{D}$, with

$$
x^{\alpha}=\prod_{\mu} x_{\mu}^{\alpha_{\mu}}, \quad \partial_{\alpha}=\prod_{\mu} \partial_{\mu}^{\alpha_{\mu}}, \quad \alpha !=\prod_{\mu} \alpha_{\mu} ! \quad \text { etc. },
$$

and we write

$$
h_{l, m}(x)=\sum_{\alpha} t_{l, m ; \alpha} x^{\alpha} .
$$


Combining eq. (3.28) with theorem 5.14 of [2] we get

$$
\sum_{\alpha} \bar{t}_{l, m ; \alpha} t_{l^{\prime}, m^{\prime} ; \alpha} \frac{\alpha !}{k_{l}}=\delta_{l, l^{\prime}} \delta_{m, m^{\prime}}
$$

with $k_{l}=2^{l} \Gamma(l+D / 2) / \Gamma(D / 2)$. This can also easily be proved starting from eq. (5.109). The decomposition of a harmonic function $f$ regular at the origin into harmonic polynomials reads

$$
f(x)=\sum_{l, m}\left(\int_{S^{D-1}} \mathrm{~d} \Omega(\hat{x}) f(\hat{x}) \bar{h}_{l, m}(\hat{x})\right) h_{l, m}(x) .
$$

With $\left.\partial^{\alpha} x^{\beta}\right|_{x=0}=\delta_{\alpha \beta} \alpha$ ! we have

$$
\left.\bar{h}_{l, m}(\partial) h_{l^{\prime}, m^{\prime}}(x)\right|_{x=0}=\sum_{\alpha} \bar{t}_{l, m ; \alpha} t_{l^{\prime}, m^{\prime} ; \alpha} \alpha !=\delta_{l l^{\prime}} \delta_{m m^{\prime}} k_{l}
$$

and thus eq. (A.123) reads

$$
f(x)=\sum_{l, m} k_{l}^{-1}\left(\bar{h}_{l m}(\partial) f(0)\right) h_{l m}(x)
$$

We also cite theorem 5.20 of [2], which states that for a harmonic homogeneous polynomial $p$ of degree $l$,

$$
p(\partial) g(r)=q_{l} r^{2-D} p\left(x / r^{2}\right)
$$

where $r=|x|$,

$$
g(r)= \begin{cases}r^{2-D} & \text { for } D=2 \\ \ln r & \text { for } D>2\end{cases}
$$

and

$$
q_{l}= \begin{cases}2^{l-1} \Gamma(l) & \text { for } D=2 \\ 2^{l} \Gamma(l+D / 2-1) / \Gamma(D / 2-1) & \text { for } D>2\end{cases}
$$

The Legendre polynomials in $D \geq 2$ dimensions are defined as the following invariants under $S O(D)$ :

$$
\sum_{m=1}^{N(l, D)} \bar{h}_{l, m}(\hat{x}) h_{l, m}(\hat{y})=\frac{2 l+D-2}{\sigma_{D}} \mathrm{P}(\hat{x} \cdot \hat{y}, l, D) .
$$

By construction, the Legendre polynomials $\mathrm{P}(z, l, D)$ are polynomials of degree $l \in \mathbb{N}$. They are often also called "Gegenbauer polynomials" and are denoted alternatively $C_{l}^{(D-2) / 2}(z)$, 
with notable differences in the normalization convention throughout the literature. A generating function is

$$
\frac{1}{D-2}\left(\frac{1}{\sqrt{1-2 h z+h^{2}}}\right)^{D-2}=\sum_{l=0}^{\infty} \mathrm{P}(z, l, D) h^{l} .
$$

This formula holds for $D \geq 3$. For $D=2$, the left side is to be replaced by $-\ln \sqrt{1-2 h z+h^{2}}$. A generalization of this formula needed in the main text is provided in theorem 2 . The Legendre polynomials have the symmetry property $\mathrm{P}(z, l, D)=$ $(-1)^{l} \mathrm{P}(-z, l, D)$, and satisfy the normalization condition

$$
\mathrm{P}(1, l, D)=\frac{(l+D-3) !}{l !(D-2) !} .
$$

For complex values of the index $\nu \in \mathbb{C}($ or $D)$, one can define an analytic continuation by means of the Gauss hypergeometric function

$$
\mathrm{P}(z, \nu, D)=\frac{\Gamma(\nu+D-2)}{\Gamma(\nu+1) \Gamma(D-1)}{ }_{2} F_{1}\left(-\nu, \nu+D-2, D / 2-1 / 2, \frac{1-z}{2}\right) .
$$

The Gauss hypergeometric function is given by the convergent expansion

$$
{ }_{2} F_{1}(a, b ; c ; x)=\sum_{n=0}^{\infty} \frac{(a)_{n}(b)_{n}}{(c)_{n} n !} x^{n}, \quad(a)_{n}=\Gamma(a+n) / \Gamma(a),
$$

for $|x|<1$. Note that the above formula has a slight anomaly in $D=2$ dimensions. Here, it gives $\mathrm{P}(\cos \alpha, \nu, 2)=\cos (\nu \alpha) / 2 \nu$ in $D=2$ dimensions for $\nu \neq 0$, and this evidently does not have a limit as $\nu \rightarrow 0$. On the other hand, the generating formula definition gives $\mathrm{P}(\cos \alpha, 0,2)=1$. The differential equation satisfied by the Legendre functions is

$$
\left(1-z^{2}\right) y^{\prime \prime}-(D-1) z y^{\prime}+\nu(\nu+D-2) y=0 .
$$

\section{B Theorems for Legendre functions}

In the main text, we use certain identities for Legendre functions in $D$ dimensions that we were not able to find in the literature, and which we therefore prove here:

Theorem 1. (Generalized Dougall's formula) For any $\nu \in \mathbb{C} \backslash \mathbb{Z}$ and $-1 \leq z \leq+1$ and $D \geq 3$, we have the identity

$$
\sum_{l=0}^{\infty} \frac{(2 l+D-2) \mathrm{P}(z, l, D)}{\nu(\nu+D-2)-l(l+D-2)}=\frac{\pi}{\sin \pi \nu} \mathrm{P}(-z, \nu, D) .
$$


Proof: For $D=3$, a proof of the theorem can be given via a contour integral argument, see [8]. We here give the following conceptually somewhat more transparent proof, valid for arbitrary $D>2$. Let $\hat{\Delta}$ be the Laplacian on the sphere $S^{D-1}$. This is an elliptic, second order partial differential operator on a compact manifold with analytic coefficients. Using standard results on the functional calculus of such operators, we can form the resolvent operator $R_{\nu}=[\hat{\Delta}+\nu(\nu+D-2)]^{-1}$ for any $\nu$ such that $\nu(\nu+D-2)$ is not an eigenvalue, i.e. $\nu \notin \mathbb{Z}$. Let $R_{\nu}(\hat{x}, \hat{y})$ be the kernel of $R_{\nu}$, which using general results on the Laplacian on compact Riemannian manifolds is known to be an analytic function on $S^{D-1} \times S^{D-1}$ apart from coincident points. Near coincident points, one has $R_{\nu} \sim[d(\hat{x}, \hat{y})]^{-(D-3) / 2}$ for $D>3$ and $R_{\nu} \sim \ln d(\hat{x}, \hat{y})$ for $D=3$, where $d(\hat{x}, \hat{y})=\arccos (\hat{x} \cdot \hat{y})$ is the geodesic distance on the sphere. A representation of $R_{\nu}$ in terms of eigenfunctions of the Laplacian is

$$
\begin{aligned}
R_{\nu}(\hat{x}, \hat{y}) & =\sum_{l=0}^{\infty} \sum_{m=1}^{N(D, l)} \frac{\bar{h}_{l, m}(\hat{x}) h_{l, m}(\hat{y})}{\nu(\nu+D-2)-l(l+D-2)} \\
& =\sigma_{D} \sum_{l=0}^{\infty} \frac{(2 l+D-2) \mathrm{P}(\hat{x} \cdot \hat{y}, l, D)}{\nu(\nu+D-2)-l(l+D-2)} .
\end{aligned}
$$

In the second line we have used the definition of the Legendre polynomials. Hence we see that the kernel $R_{\nu}$ is, up to a constant, precisely equal to the left side of the Dougall formula.

By definition, the kernel obeys $[\hat{\Delta}+\nu(\nu+D-2)] R_{\nu}=\delta$ in the sense of distributions. However, since $R_{\nu}$ is evidently invariant under $S O(D)$-transformations, we may write $R_{\nu}(\hat{x}, \hat{y})=y(z)$ for some analytic function of $z=\hat{x} \cdot \hat{y}$ when $z \neq 1$. As a consequence of the differential equation satisfied by $R_{\nu}$, it can easily be seen that $y$ satisfies the differential equation for the Legendre function of dimension $D$ and degree $\nu$, see eq. (A.134). Hence we have

$$
y(z)=A \mathrm{P}(z, \nu, D)+B \mathrm{P}(-z, \nu, D)
$$

for some $A, B \in \mathbb{C}$ as $\mathrm{P}(z, \nu, D), \mathrm{P}(-z, \nu, D)$ span the solution space of eq. (A.134). Furthermore, $\mathrm{P}(-z, \nu, D)$ is singular at $z=1$ and regular at $z=-1$ (see e.g. [8]), as is $y(z)$. By contrast, $\mathrm{P}(z, \nu, D)$ is singular at $z=-1$ and regular at $z=1$. Thus, we must have $A=0$ in eq. (B.137).

In order to determine the constant $B$, we evaluate $y(-1)$. Using $\mathrm{P}(-1, l, D)=$ $(-1)^{l} \mathrm{P}(1, l, D)$ and the formula (A.131), we find using various summation identities for the Gamma-function:

$$
\begin{aligned}
\frac{1}{\sigma_{D}} y(-1) & =\frac{1}{(D-2) !} \sum_{l=0}^{\infty}(-1)^{l}\left(\frac{1}{\nu-l}-\frac{1}{\nu+l+D-2}\right) \frac{(l+D-3) !}{l !} \\
& =\frac{\Gamma(-\nu) \Gamma(\nu+D-2)}{\Gamma(D-1)}=\frac{\Gamma(\nu+D-2)}{\Gamma(\nu+1) \Gamma(D-1)} \frac{\pi}{\sin \pi \nu} .
\end{aligned}
$$

Comparing this with the normalization of $\mathrm{P}(-z, \nu, D)$ at $z=-1$, we get the statement of the theorem. 
The next theorem is a generalization of formula (A.130).

Theorem 2. (Shifted generating functional formula) For any $\delta \in \mathbb{C} \backslash \mathbb{Z}$ and $-1 \leq z \leq$ $+1,|h|<1$ and even $D \geq 4$, we have the identity

$$
\begin{aligned}
& \sum_{l=0}^{\infty} h^{l} \mathrm{P}(z, l+\delta, D)=\left(2 \delta_{D}\right)^{-1} A_{D}\left\{\left(z+\mathrm{i} \operatorname{sqrt} 1-z^{2}\right)^{\delta_{D}}{ }_{2} F_{1}\left(\delta_{D}, 1 ; \delta_{D}+1 ; h\left(z+\mathrm{i} \text { sqrt } 1-z^{2}\right)\right)\right. \\
& \left.+\left(z-\mathrm{i} \operatorname{sqrt} 1-z^{2}\right)^{\delta_{D}}{ }_{2} F_{1}\left(\delta_{D}, 1 ; 1+\delta_{D}, h\left(z-\mathrm{i} \operatorname{sqrt} 1-z^{2}\right)\right)\right\},
\end{aligned}
$$

where $\delta_{D}$ and the differential operator $A_{D}$ are given by

$$
\begin{aligned}
A_{D} & =\frac{1}{\Gamma(D / 2)}\left(\frac{\partial}{2 h \partial z}\right)^{(D-2) / 2} \\
\delta_{D} & =\delta+(D-2) / 2
\end{aligned}
$$

For $D=2$ the differential operator is missing. For odd $D \geq 5$, we have the formula

$$
\begin{aligned}
& \sum_{l=0}^{\infty} h^{l} \mathrm{P}(z, l+\delta, D)=A_{D}\left\{\frac{-1}{\sqrt{1+h^{2}+2 h z}} \times\right. \\
& \left.\left(F_{1}\left(-\delta_{D}, \delta_{D}, 1,1 ; \frac{1-z}{2}, \frac{1-t_{-}}{2}\right)+\frac{z-t_{-}}{2} F_{1}\left(1-\delta_{D}, 1+\delta_{D}, 1,2 ; \frac{1-z}{2}, \frac{1-\tau\left(t_{+}\right)}{2}\right)\right)\right\} .
\end{aligned}
$$

where this time

$$
\begin{aligned}
A_{D} & =\frac{\sqrt{\pi}}{2 \Gamma(D / 2)}\left(\frac{\partial}{2 h \partial z}\right)^{(D-3) / 2} \\
\delta_{D} & =\delta+(D-3) / 2
\end{aligned}
$$

For $D=3$ the differential operator is missing. $F_{1}$ is the two-variable generalization of the hypergeometric function defined by

$$
F_{1}(a, b, c ; d ; x, y)=\sum_{m=0}^{\infty} \sum_{n=0}^{\infty} \frac{(a)_{m+n}(b)_{m}(c)_{n}}{(d)_{m+n} m ! n !} x^{m} y^{n}
$$

and we have defined $t_{ \pm}=h^{-1}\left(1 \pm \sqrt{1+h^{2}-2 h z}\right)$, and $\tau(t)$ by eq. (B.155).

Remark: There is an apparent asymmetry in the formulas for even and odd $D$. One is tempted to believe that both formulas given for the shifted generating function are valid for 
all $D$ (when appropriately interpreted), but we have not been able to show this. The first formula may further be rewritten noting the standard formula

$$
f(\delta, x)=(2 \delta)^{-1}{ }_{2} F_{1}(\delta, 1 ; \delta+1 ; 1-x)=\sum_{n=0}^{\infty} \frac{(\delta)_{n}}{n !}[\psi(n+1)-\psi(\delta+n)-\ln x] x^{n} .
$$

In the main text, we use the theorem to calculate the sums eq. (4.71) for $D=2$ and eq. (4.93) for $D=3$, where we also make use of the relation

$$
\mathrm{P}(z, \nu, D)=(-1)^{D-3} \mathrm{P}(z,-\nu-D+2, D) .
$$

We then apply the recurrence relation (B.146) to obtain $g_{D}(\delta, \cos \beta, t)$ as in eq. (4.94) (for even $D)$.

Proof for even $D$ : For $D=2$, the proof of the theorem follows immediately from the definition of the hypergeometric function. The alternate form (B.144) is obtained from transformation formula 15.3 .10 of [1] for hypergeometric functions. For $D$ even and $D>2$, we prove the formula using the recurrence identity

$$
\frac{\mathrm{d}}{\mathrm{d} z} \mathrm{P}(z, \nu, D)=D \mathrm{P}(z, \nu-1, D+2) .
$$

Proof for odd $D$ : For odd $D$, we proceed using the same recurrence identity, but in order to be able to do so, we have to evaluate $\sum_{l=(D-3) / 2}^{\infty} \mathrm{P}(z, l+\delta, 3)$, and this requires some extra work. We start with the Schlaefli integral formula for Legendre functions [28],

$$
P(z, \nu, 3)=\frac{1}{2 \pi \mathrm{i}} \oint_{C^{+}} \frac{\left(t^{2}-1\right)^{\nu}}{2^{\nu}(t-z)^{\nu+1}} \mathrm{~d} t .
$$

To make $\left(t^{2}-1\right)^{\nu} 2^{-\nu}(t-z)^{-\nu-1}$ single-valued, we have to introduce two cuts in the complex plane, and we follow [26] choosing these cuts as the half-line $\gamma_{1}=(-\infty,-1)$ and a curve $\gamma_{2}$ joining the points $t=1$ and $t=z$, parametrized by

$$
\frac{1+\eta z}{\eta+z} \quad(1 \leq \eta<\infty)
$$

The contour $C^{+}$encircles $\gamma_{2}$ counterclockwise, see fig. 8. In [26], this particular representation of Legendre functions made it possible to determine derivatives of Legendre functions with respect to their degree $\nu$.

We set $\delta_{D}=\delta+(D-3) / 2$ and have

$$
\begin{aligned}
\sum_{l=(D-3) / 2}^{\infty} h^{l} P\left(z, l+\delta_{D}, 3\right) & =\sum_{l=0}^{\infty} h^{l} P\left(z, l+\delta_{D}, 3\right) \\
& =\frac{1}{2^{1+\delta_{D}} \pi \mathrm{i}} \oint_{C^{+}} \mathrm{d} t\left(\frac{t^{2}-1}{t-z}\right)^{\delta_{D}} \frac{1}{t-z-h\left(t^{2}-1\right) / 2} \\
& =\frac{1}{2^{1+\delta_{D}} \pi \mathrm{i}} \oint_{C^{+}} \mathrm{d} t \chi(t) \frac{1}{t-z-h\left(t^{2}-1\right) / 2}
\end{aligned}
$$




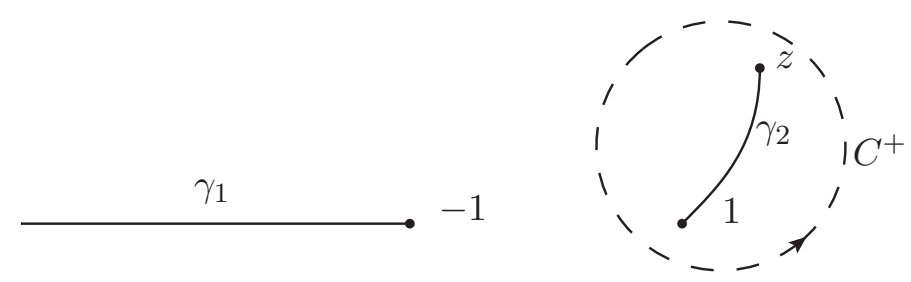

Figure 8: Cuts chosen to make $\left(t^{2}-1\right)^{\delta}(t-z)^{-\delta}$ well-defined.

where in the first equation, we have interchanged the order of summation and integration, and in the second we have set $\chi(t)=\left(t^{2}-1\right)^{\delta_{D}}(t-z)^{-\delta_{D}}$ (defined with the same cuts as above). Our aim will be to express $\chi$ as a sum of two functions $\chi_{1}, \chi_{2}$ that have cuts on $\gamma_{1}$ and $\gamma_{2}$ respectively, and are analytic elsewhere. Then we will be able to carry out the integration in eq. (B.149). Assuming $\Re \delta_{D}<0$, we apply formula 3.1 .11 of [17],

$$
\chi(t)=-\pi^{-1} \int \mathrm{d} w \mathrm{~d} \bar{w}(\bar{\partial} \chi(w, \bar{w}))(w-t)^{-1} .
$$

We introduce a function $\rho_{1}$ that equals 1 in a small neighborhood of the cut $(-\infty, 1)$ and 0 outside a slightly bigger neighborhood. Also, we introduce $\rho_{2}$, smooth, equal to 1 in a small neighborhood of the cut (B.148) and 0 in a slightly bigger neighborhood, so that $\operatorname{supp} \rho_{1} \cap \operatorname{supp} \rho_{2}=\emptyset$. As $\chi$ is analytic away from the cuts (i.e. $\bar{\partial} \chi=0$ on $\mathbb{C} \backslash\left(\gamma_{1} \cup \gamma_{2}\right)$ ), we can modify eq. (B.150) in the following way,

$$
\begin{aligned}
\chi(t) & =\chi_{1}(t)+\chi_{2}(t), \\
\chi_{i}(t) & =-\pi^{-1} \int \mathrm{d} w \mathrm{~d} \bar{w}(\bar{\partial} \chi(w, \bar{w}))(w-t)^{-1} \rho_{i}(w, \bar{w}), \quad(i=1,2) .
\end{aligned}
$$

Now by theorem 3.1 .12 of $[17]$

$$
\begin{aligned}
\chi_{1}(t) & =\frac{1}{2 \pi \mathrm{i}} \int_{-\infty}^{-1} \mathrm{~d} x(\chi(x+\mathrm{i} 0)-\chi(x-\mathrm{i} 0))(x-t)^{-1} \\
& =\frac{\sin \delta_{D} \pi}{\pi} \int_{-\infty}^{-1} \mathrm{~d} x\left(\frac{x^{2}-1}{z-x}\right)_{D}^{\delta}(x-t)^{-1}
\end{aligned}
$$

We substitute $u:=2 /(1-x)$ and obtain

$$
\begin{aligned}
\chi_{1}(t) & =-\frac{\sin \delta_{D} \pi}{\pi} 2^{\delta_{D}} \int_{0}^{1} u^{-1-\delta_{D}}(1-u)_{D}^{\delta}\left(1-\frac{1-z}{2} u\right)^{-\delta_{D}}\left(1-\frac{1-t}{2} u\right)^{-1} \\
& =-\frac{\sin \delta_{D} \pi}{\pi} 2^{\delta_{D}} B\left(1+\delta_{D},-\delta_{D}\right) F_{1}\left(-\delta_{D}, \delta_{D}, 1,1 ;(1-z) / 2,(1-t) / 2\right)
\end{aligned}
$$


where in the second equation, we have used formula 3.211 of $[12] ; F_{1}$ is the hypergeometric function of two variables defined above, and $B$ is the Beta function $B(x, y)=\Gamma(x) \Gamma(y) / \Gamma(x+$ $y)$. We next determine $\chi_{2}$,

$$
\begin{aligned}
\chi_{2}(t) & =-\pi^{-1} \int \mathrm{d} w \mathrm{~d} \bar{w}(\partial g)(w-t)^{-1} \rho_{2}(w, \bar{w}) \\
& =-\pi^{-1} \int \mathrm{d} \tau \mathrm{d} \bar{\tau} \frac{\partial w}{\partial \tau} \frac{\partial \bar{w}}{\partial \bar{\tau}}\left(\frac{\partial \bar{\tau}}{\partial \bar{w}} \partial_{\bar{\tau}} \chi(w(\tau))\right)(w(\tau)-t)^{-1} \rho_{2}(w(\tau), \bar{w}(\bar{\tau})) \\
& =-\frac{1}{2 \pi \mathrm{i}} \int_{-\infty}^{-1} d x(\chi(x+\mathrm{i} 0)-\chi(x-\mathrm{i} 0)) \frac{1-z^{2}}{(z-x)^{2}}\left(\frac{1-z x}{z-x}-t\right)^{-1} \\
& =-\frac{\sin \delta_{D} \pi}{\pi} \int_{-\infty}^{-1} \mathrm{~d} x\left(\frac{x^{2}-1}{z-x}\right)_{D}^{\delta} \frac{1-z^{2}}{(z-x)^{2}}\left(\frac{1-z x}{z-x}-t\right)^{-1}
\end{aligned}
$$

where in the second equation we have performed a change of coordinates,

$$
\tau(w)=\frac{1-w z}{z-w}
$$

with inverse $w(\tau)=(1-\tau z) /(z-\tau)$. This coordinate transformation maps $\gamma_{1}$ on $\gamma_{2}$ and vice versa. Again substituting $u=2 /(1-x)$, we get

$$
\begin{aligned}
\chi_{2}(t) & =-\frac{\sin \delta_{D} \pi}{\pi} \frac{1-z^{2}}{z-t} 2^{-1+\delta_{D}} \int_{0}^{1} \mathrm{~d} u u^{-\delta_{D}}(1-u)^{\delta_{D}}\left(\frac{1-z}{2} u\right)^{-1-\delta_{D}}\left(\frac{1-\tau(t)}{2} u\right)^{-1} \\
& =\frac{\sin \delta_{D} \pi}{\pi} \frac{1-z^{2}}{t-z} 2^{-1+\delta_{D}} \\
& \times B\left(1+\delta_{D}, 1-\delta_{D}\right) F_{1}\left(1-\delta_{D}, 1+\delta_{D}, 1,2 ;(1-z) / 2,(1-\tau(t)) / 2\right)
\end{aligned}
$$

In eq. (B.149), we replace

$$
\begin{aligned}
\frac{1}{t-z-h\left(t^{2}-1\right) / 2} & =-\frac{2}{h\left(t-t_{+}\right)\left(t-t_{-}\right)} \\
t_{ \pm} & =h^{-1}\left(1 \pm \sqrt{1+h^{2}-2 h z}\right) .
\end{aligned}
$$

For $-1 \leq z \leq 1, t_{+} \in \gamma_{1}$ and $t_{-} \in \gamma_{2}$, so $t_{-}$lies inside $C^{+}$and $t^{+}$outside. We can now calculate the contribution of $\chi_{1}(t)$ to the integral (B.149),

$$
\frac{1}{2^{1+\delta_{D}} \pi \mathrm{i}} \oint_{C^{+}} \mathrm{d} t \chi_{1}(t) \frac{1}{t-z-h\left(t^{2}-1\right) / 2}
$$

which is now a simple residue integral. We obtain

$$
\frac{\sin \delta_{D} \pi}{\pi} \frac{1}{\sqrt{1+h^{2}+2 h z}} B\left(1+\delta_{D},-\delta_{D}\right) F_{1}\left(-\delta_{D}, \delta_{D}, 1,1 ;(1-z) / 2,\left(1-t_{-}\right) / 2\right) .
$$


In a similar manner, we calculate the contribution of $\chi_{2}$,

$$
\begin{aligned}
& \frac{B\left(1+\delta_{D}, 1-\delta_{D}\right) \sin \pi \delta_{D}\left(1-z^{2}\right)}{4 \pi^{2} \mathrm{i}} \\
\times & \oint_{D^{-}} \mathrm{d} \tau\left(\frac{1-z^{2}}{(z-\tau)^{2}} F_{1}\left(1-\delta_{D}, 1+\delta_{D}, 1,2 ;(1-z) / 2,(1-\tau) / 2\right) \frac{1}{t(\tau)-z}\right. \\
\times & \left.\left(-\frac{2}{h}\right) \frac{z-\tau}{\left(t_{-}-z\right)\left(\tau-\tau\left(t_{-}\right)\right.} \frac{z-\tau}{\left(t_{+}-z\right)\left(\tau-\tau\left(t_{+}\right)\right.}\right),
\end{aligned}
$$

where we used the coordinate transformation (B.155). The contour $D^{-}$is the image of $C^{+}$ under this transformation. $D^{-}$encircles $\gamma_{2}$ and runs clockwise, not crossing the cuts $\gamma_{1}, \gamma_{2}$. We can deform $D^{-}$into $C^{-}$by which we mean the contour $C^{+}$with negative orientation. $\tau\left(t_{+}\right)$is on the inside of $C^{-}, \tau\left(t_{-}\right)$on the outside. Thus the residue integral (B.160) is

$$
\begin{aligned}
& \frac{\sin \pi \delta_{D}}{\pi} \frac{1}{\sqrt{1+h^{2}-2 h z}} \frac{1-z^{2}}{2\left(z-t_{+}\right)} \\
& \times \quad B\left(1+\delta_{D}, 1-\delta_{D}\right) F_{1}\left(1-\delta_{D}, 1+\delta_{D}, 1,2 ;(1-z) / 2,\left(1-\tau\left(t_{+}\right)\right) / 2\right) .
\end{aligned}
$$

Putting together eqs. (B.159) and (B.161), we obtain

$$
\begin{aligned}
\sum_{l=0}^{\infty} h^{l} P\left(z, l+\delta_{D}, 3\right) & =\frac{\sin \delta_{D} \pi}{\pi} \frac{1}{\sqrt{1+h^{2}+2 h z}} \\
& \times\left(B\left(1+\delta_{D},-\delta_{D}\right) F_{1}\left(-\delta_{D}, \delta_{D}, 1,1 ;(1-z) / 2,\left(1-t_{-}\right) / 2\right)\right. \\
& +\frac{1-z^{2}}{2\left(z-t_{+}\right)} B\left(1+\delta_{D}, 1-\delta_{D}\right) \\
& \left.\times F_{1}\left(1-\delta_{D}, 1+\delta_{D}, 1,2 ;(1-z) / 2,\left(1-\tau\left(t_{+}\right)\right) / 2\right)\right)
\end{aligned}
$$

Above, we have used $\Re \delta_{D}<0$. However both sides of eq. (B.162) are analytic in $\delta_{D}$ throughout the complex plane for $|h|<1$ (with possible exceptions for $\delta_{D} \in \mathbb{Z}$ ). This means that they are identical and eq. (B.162) must hold for $\Re \delta \geq 0$ as well. Eq. (B.141) follows from the first line of eq. (B.149), the recurrence formula (B.146), standard identities for the gamma function and the relation $\left(z-t_{+}\right)\left(z-t_{-}\right)=z^{2}-1$.

We finally mention another representation of the Legendre functions used in the main text:

Theorem 3. For $\nu \in \mathbb{C} \backslash \mathbb{Z},|z|<1$ we have the formula

$$
\mathrm{P}(z, \nu, D)=\frac{\sin \pi \nu}{\pi} \frac{2^{-(D+1) / 2}}{\Gamma(D / 2)} \sum_{n=0}^{\infty}(-2 z)^{n} \frac{\Gamma(-\nu / 2+n / 2) \Gamma(\nu / 2+n / 2+D / 2-1)}{n !} .
$$


Proof: We prove this first for $D=2$. Let $z=\cos \alpha$. We have the identities

$$
\begin{aligned}
\cos \nu \alpha= & { }_{2} F_{1}\left(-\nu / 2, \nu / 2 ; 1 / 2 ; \sin ^{2} \alpha\right) \\
= & \frac{\pi}{\Gamma(-\nu / 2+1 / 2) \Gamma(\nu / 2+1 / 2)}{ }_{2} F_{1}\left(-\nu / 2, \nu / 2 ; 1 / 2 ; \cos ^{2} \alpha\right) \\
& -2 \cos \alpha \frac{\pi}{\Gamma(-\nu / 2) \Gamma(\nu / 2)}{ }_{2} F_{1}\left(-\nu / 2+1 / 2, \nu / 2+1 / 2 ; 3 / 2 ; \cos ^{2} \alpha\right),
\end{aligned}
$$

where in the second line we have used a standard transformation formula for hypergeometric functions. We now use $\mathrm{P}(\cos \alpha, \nu, 2)=\cos (\nu \alpha) / 2 \nu$, and we expand the hypergeometric series in the second and third line, using the doubling identity of the Gamma function, $\sqrt{\pi} \Gamma(2 x)=2^{2 x-1} \Gamma(x) \Gamma(x+1 / 2)$, in various ways. Then we obtain the statement of the theorem for $D=2$. The case $D=3$ is covered by formula 8.1.4 of [1], together with the use of the doubling identity as above.

For general $D \in \mathbb{N}$, we use the recurrence formula (B.146), combined with a standard formula for the derivatives of the hypergeometric function. This then gives the formula for all even $D$ starting from $D=2$ and all odd $D$ starting from $D=3$.

\section{The free field vertex operators}

The non-vanishing partial derivatives of the basic field $\varphi$ in the theory defined by the Schwinger functions eq. (3.30) are

$$
\varphi^{l, m}=c_{l}^{-1} \bar{t}_{l, m ; \alpha} \partial^{\alpha} \varphi
$$

where $c_{l}$ will be chosen later. The composite fields are labeled by multiindices

$$
\mathcal{O}_{a}=(a !)^{-1 / 2} \prod_{l, m}\left(\varphi^{l, m}\right)^{a_{l, m}}
$$

We use Latin letters for the multiindices denoting composite fields and Greek letters for

multiindices when dealing with polynomials in $x$ or $\partial$. The basic field $\varphi$ is harmonic by the field equation (3.27), so we may use eq. (A.125),

$$
\varphi(x)=\sum_{l, m} \frac{c_{l}}{k_{l}} h_{l, m}(x) \varphi^{l, m}(0)
$$

This has to be understood as an equation for insertions into Schwinger functions. Now the OPE of $\varphi$ with a field $\mathcal{O}_{a}$ can easily be deduced from eq. (3.30) and the definition of $\varphi^{l, m}$, eq. (C.165):

$$
\begin{aligned}
\varphi(x) \mathcal{O}_{a}(0) & =\sum_{l, m} \frac{c_{l}}{k_{l}} h_{l, m}(x)\left(\varphi^{l, m} \mathcal{O}_{a}\right)(0) \\
& +\sum_{l, m} c_{l}^{-1} \bar{h}_{l, m}(\partial) g(r) \frac{\partial \mathcal{O}_{a}}{\partial \varphi^{l, m}}(0)
\end{aligned}
$$


where $r=|x|$ as always; see eq. (A.127) for the definition of the "Euclidean propagator" $g$. Again, this has to be understood as an equation for insertions:

$$
\left\langle\varphi(x) \mathcal{O}_{a}(0) \varphi\left(x_{1}\right) \ldots \varphi\left(x_{s}\right)\right\rangle=\left\langle(\text { RHS of eq. }(\mathrm{C} .168)) \varphi\left(x_{1}\right) \ldots \varphi\left(x_{s}\right)\right\rangle
$$

If we write down the left hand side of eq. (C.169) as in eq. (3.31), where the points $x$ and 0 are represented by vertices $v$ and $w$ respectively $\left(x_{v}=x, x_{w}=0\right)$, then the first term of the right hand side in eq. (C.168) can be understood as a Taylor expansion (in $x$ ) of those graphs without a line $(v w)$, and the second term as the Taylor expansion of graphs including that line. In order for these Taylor expansions to converge, we must have $\left|x_{1}\right|, \ldots,\left|x_{s}\right|>|x|$. Using eqs. (A.126) and (C.168) we obtain

$$
\begin{aligned}
\varphi(x) \mathcal{O}_{a}(0) & =\sum_{l, m} \sqrt{a_{l, m}+1} \frac{c_{l}}{k_{l}} h_{l, m}(x) \mathcal{O}_{a+e_{(l, m)}}(0) \\
& +\sum_{l, m} \sqrt{a_{l, m}} \frac{q_{l}}{c_{l}} \bar{h}_{l, m}(x) r^{-2 l-D+2} \mathcal{O}_{a-e_{(l, m)}}(0)
\end{aligned}
$$

where by $e_{(l, m)}$, we mean the multiindex defined by $\left(e_{(l, m)}\right)_{l^{\prime}, m^{\prime}}=\delta_{l, l^{\prime}} \delta_{m, m^{\prime}}$, and we define $\left(a-e_{(l, m)}\right):=0$ for $a_{l, m}=0$. To obtain a symmetric form of the OPE, we choose

$$
c_{l}=\sqrt{q_{l} k_{l}}= \begin{cases}2^{l} \Gamma(l) \sqrt{l / 2} & \text { for } D=2 \\ 2^{l} \Gamma(l+D / 2-1) \sqrt{2(l+D / 2-1) /(D-2)} & \text { for } D>2 .\end{cases}
$$

We introduce the abstract vector space $V$ spanned by the field labels $a$ and creation and annihilation operators $\mathbf{b}_{l, m}^{+}, \mathbf{b}_{l, m}$ on $V$ by

$$
\begin{aligned}
& \mathbf{b}_{l, m}^{+} a=\sqrt{a_{l, m}+1}\left(a+e_{(l, m)}\right) \\
& \mathbf{b}_{l, m} a=\sqrt{a_{l, m}}\left(a-e_{(l, m)}\right) .
\end{aligned}
$$

We also introduce the vertex operator $Y_{0}(\varphi, x)$ that corresponds to a multiplication of an insertion with the free field $\varphi(x)$. We rewrite the left-hand side of eq. (C.170) in this notation by

$$
Y_{0}(\varphi, x) a
$$

and we can read off the right hand side of eq. (C.170) that

$$
Y_{0}(\varphi, x)=K_{D} \sum_{l, m} \frac{1}{\sqrt{\omega(D, l)}}\left(h_{l, m}(x) \mathbf{b}_{l, m}^{+}+\bar{h}_{l, m}(x) r^{-2 l-D+2} \mathbf{b}_{l, m}\right)
$$

with $K_{D}=1$ for $D=2, K_{D}=\sqrt{D-2}$ for $D>2$ and $\omega(D, l)=2 l+D-2$.

That the vertex operators for composite fields $Y_{0}(a, x)$ are given by eq. (3.35) is evident from the remarks on how to determine the Schwinger functions of composite operators from those 
of the basic field below eq. (3.30).

In the main text, we also used Wick's theorem, which we cite for completeness in the following form: Let $\mathbf{b}_{l, m}^{\#} \mathbf{b}_{l^{\prime}, m^{\prime}}^{\#}$ denote the "contraction" between two creation and/or annihilation operators, defined by

$$
\mathbf{b}_{l, m}^{\#} \mathbf{b}_{l^{\prime}, m^{\prime}}^{\#}=: \mathbf{b}_{l, m}^{\#} \mathbf{b}_{l^{\prime}, m^{\prime}}^{\#}:+\mathbf{b}_{l, m}^{\#} \mathbf{b}_{l^{\prime}, m^{\prime}}^{\#} .
$$

Writing $\mathbf{b}_{i}^{\#}$ for $\mathbf{b}_{l_{i}, m_{i}}^{\#}$ and $\mathbf{b}_{i^{\prime}}^{\#}$ for $\mathbf{b}_{l_{i}^{\prime}, m_{i}^{\prime}}^{\#}$, we have the combinatoric identity

$$
\begin{aligned}
: \mathbf{b}_{1}^{\#} \ldots \mathbf{b}_{n}^{\#}:: \mathbf{b}_{1^{\prime}}^{\#} \ldots \mathbf{b}_{p^{\prime}}^{\#}: & =\sum_{s=0}^{\min (n, p)} \sum_{\substack{i_{1}<\ldots<i_{s} \\
j_{1} \neq \ldots \neq j_{s}}} \mathbf{b}_{i_{1}}^{\#} \mathbf{b}_{j_{1}^{\prime}}^{\#} \ldots \mathbf{b}_{i_{s}}^{\#} \mathbf{b}_{j_{s}^{\prime}}^{\#} \\
& \times: \mathbf{b}_{1}^{\#} \ldots \mathbf{b}_{n}^{\#} \mathbf{b}_{1^{\prime}}^{\#} \ldots \mathbf{b}_{p^{\prime}}^{\#}:_{\left(i_{1}, \ldots, i_{s} ; j_{1}, \ldots, j_{s}\right)}^{\#}
\end{aligned}
$$

where double dots mean normal ordering and the subscript $\left(i_{1}, \ldots, i_{s} ; j_{1}, \ldots, j_{s}\right)$ means that the respective creation or annihilation operators have been removed from the normal ordered product.

\section{References}

[1] M. Abramowitz and I.A. Stegun. Handbook of Mathematical Functions with Formulas, Graphs, and Mathematical Tables. Dover, New York, 1964.

[2] S. Axler, P. Bourdon, and W. Ramey. Harmonic Function Theory. Springer, New York, 2001.

[3] R. E. Borcherds. Vertex algebras, Kac-Moody algebras, and the monster. Proc. Nat. Acad. Sci., 83:3068-3071, 1986.

[4] R. E. Borcherds. Vertex algebras. Topological field theory, primitive forms and related topics, 1997.

[5] D. Brydges, J. Dimock, and T. R. Hurd. Applications of the renormalization group. Prepared for Mathematical Quantum Theory Conference, Vancouver, Canada, 4-8 Aug 1993.

[6] K. G. Chetyrkin, A. L. Kataev, and F. V. Tkachov. New Approach to Evaluation of Multiloop Feynman Integrals: The Gegenbauer Polynomial x Space Technique. Nucl. Phys., B174:345-377, 1980. 
[7] A. Connes and D. Kreimer. Renormalization in quantum field theory and the RiemannHilbert problem. I: The Hopf algebra structure of graphs and the main theorem. Commun. Math. Phys., 210:249, 2000.

[8] A. Erdélyi. Higher Transcendental Functions, Vol.1. McGraw-Hill, 1953.

[9] I. Frenkel, J. Lepowsky, and A. Meurman. Vertex Operator Algebras and the Monster. Boston, USA: Academic (1988) 508 P. (Pure and Apllied Mathematics, 134).

[10] B. Fuglede. Integration on the $n$-th power of a hyperbolic space in terms of invariants under diagonal action of isometries (Lorentz transformations). Commun. Math. Phys., 129:481-509, 1990.

[11] M. R. Gaberdiel. An introduction to conformal field theory. Rept.Prog.Phys., 63:607$667,2000$.

[12] I.S. Gradshteyn and I.M. Ryzhik. Table of Integrals, Series and Products. Academic Press, New York, 1965.

[13] J. Holland. Construction of operator product expansion coefficients via consistency conditions. Diplomarbeit, Universität Göttingen, 2008.

[14] S. Hollands. Quantum field theory in terms of consistency conditions I: General framework, and perturbation theory via Hochschild cohomology. arXiv:0802.2198, 2008.

[15] S. Hollands, C. Kopper, and H. Olbermann. In preparation.

[16] S. Hollands and R.M. Wald. Axiomatic quantum field theory in curved spacetimes. arXiv:0803.2003 [gr-qc], 2008.

[17] L. Hörmander. The Analysis of Linear Partial Differential Operators I. Springer, 1983.

[18] A. Jaffe and J. Glimm. Constuctive Quantum Field Theory. Collected Papers Vol.2. Birkhäuser Boston, 1985.

[19] V. Kac. Vertex Algebras for Beginners. AMS, Providence, Rhode Island, 1997.

[20] G. Keller and C. Kopper. Perturbative renormalization of composite operators via flow equations. 2. Short distance expansion. Commun. Math. Phys., 153:245-276, 1993.

[21] G. Keller, C. Kopper, and M. Salmhofer. Perturbative renormalization and effective Lagrangians in $\phi^{4}$ in four-dimensions. Helv. Phys. Acta, 65:32-52, 1992.

[22] K.J. Keller. Euclidean Epstein-Glaser renormalization. arXiv:0902.4789 [math-ph], 2009. 
[23] J. Magnen and V. Rivasseau. Constructive $\phi^{4}$ field theory without tears. Annales Henri Poincare, 9:403-424, 2008.

[24] N. M. Nikolov. Vertex algebras in higher dimensions and global conformally invariant quantum field theory. Commun. Math. Phys., 253:283-322, 2004.

[25] V. Rivasseau. From perturbative to constructive renormalization. Princeton, USA: Univ. Pr. (1991) 336 p. (Princeton series in physics).

[26] R. Szmytkowski. On the derivative of the Legendre function of the first kind with respect to its degree. J. Phys. A: Math. Gen., 39:1514715172, 2006.

[27] S. Weinzierl. The art of performing loop integrals. arXiv:hep-ph/0604068.

[28] E. T. Whittaker and G. N. Watson. A Course of Modern Analysis. Cambridge University Press, fourth edition, 1927. Reprinted 1990.

[29] K. G. Wilson. Nonlagrangian models of current algebra. Phys. Rev., 179:1499-1512, 1969.

[30] K. G. Wilson and W. Zimmermann. Operator product expansions and composite field operators in the general framework of quantum field theory. Commun. Math. Phys., 24:87-106, 1972.

[31] J. Zinn-Justin. Quantum Field Theory and Critical Phenomena. Oxford University Press, 1993. 